\title{
Hybrid extragradient method for hierarchical variational inequalities
}

\author{
Lu-Chuan Ceng ${ }^{1,2}$, Abdul Latif ${ }^{3 *}$, Qamrul Hasan Ansari ${ }^{4,5}$ and Jen-Chih Yao ${ }^{6,3}$
}

"Correspondence: alatif@kau.edu.sa ${ }^{3}$ Department of Mathematics, King Abdulaziz University, P.O. Box 80203 Jeddah, 21589, Saudi Arabia Full list of author information is available at the end of the article

\begin{abstract}
In this paper, we consider a hierarchical variational inequality problem (HVIP) defined over a common set of solutions of finitely many generalized mixed equilibrium problems, finitely many variational inclusions, a general system of variational inequalities, and the fixed point problem of a strictly pseudocontractive mapping. By combining Korpelevich's extragradient method, the viscosity approximation method, the hybrid steepest-descent method and Mann's iteration method, we introduce and analyze a multistep hybrid extragradient algorithm for finding a solution of our HVIP. It is proven that under appropriate assumptions, the proposed algorithm converges strongly to a solution of a general system of variational inequalities defined over a common set of solutions of finitely many generalized mixed equilibrium problems (GMEPs), finitely many variational inclusions, and the fixed point problem of a strictly pseudocontractive mapping. In the meantime, we also prove the strong convergence of the proposed algorithm to a unique solution of our HVIP. The results obtained in this paper improve and extend the corresponding results announced by many others. MSC: 49J30; 47H09; 47J20

Keywords: hierarchical variational inequalities; multistep hybrid extragradient algorithm; general system of variational inequalities; generalized mixed equilibrium problem; variational inclusions; strictly pseudocontractive mappings; nonexpansive mappings
\end{abstract}

\section{Introduction and formulations}

\subsection{Variational inequalities and equilibrium problems}

Let $C$ be a nonempty closed convex subset of a real Hilbert space $H$ and $A: C \rightarrow H$ be a nonlinear mapping. A variational inequality problem (VIP) is to find a point $x \in C$ such that

$$
\langle A x, y-x\rangle \geq 0, \quad \forall y \in C .
$$

The solution set of the VIP (1.1) defined by $C$ and $A$ is denoted by $\operatorname{VI}(C, A)$. The theory of variational inequalities is a well established subject in nonlinear analysis and optimization. For different aspects of variational inequalities and their generalizations, we refer to [1-3] and the references therein. Several solution methods for solving different kinds of variational inequality have appeared in literature. Korpelevich's extragradient method [4] is one of them. It is further studied in [5].

○2014 Ceng et al.; licensee Springer. This is an Open Access article distributed under the terms of the Creative Commons Attribution License (http://creativecommons.org/licenses/by/2.0), which permits unrestricted use, distribution, and reproduction in any medium, provided the original work is properly cited. 
Let $\Theta: C \times C \rightarrow \mathbf{R}$ be a bifunction. The equilibrium problem (EP) is to find $x \in C$ such that

$$
\Theta(x, y) \geq 0, \quad \forall y \in C
$$

The set of solutions of EP is denoted by $\operatorname{EP}(\Theta)$. It is a unified model of several problems, namely, variational inequalities, Nash equilibrium problems, optimization problems, saddle point problems, etc. For further details of EP, we refer to [6, 7] and the references therein.

Let $\varphi: C \rightarrow \mathbf{R}$ be a real-valued function and $A: H \rightarrow H$ be a nonlinear mapping. The generalized mixed equilibrium problem (GMEP) [8] is to find $x \in C$ such that

$$
\Theta(x, y)+\varphi(y)-\varphi(x)+\langle A x, y-x\rangle \geq 0, \quad \forall y \in C .
$$

We denote the set of solutions of $\operatorname{GMEP}(1.3)$ by $\operatorname{GMEP}(\Theta, \varphi, A)$. The $\operatorname{GMEP}(1.3)$ is very general in the sense that it includes, as special cases, optimization problems, variational inequalities, minimax problems, Nash equilibrium problems in noncooperative games, and others. The GMEP is further considered and studied in $[9,10]$ and the references therein.

When $A \equiv 0$, then GMEP (1.3) reduces to the following mixed equilibrium problem (MEP): find $x \in C$ such that

$$
\Theta(x, y)+\varphi(y)-\varphi(x) \geq 0, \quad \forall y \in C .
$$

The set of solutions of MEP is denoted by $\operatorname{MEP}(\Theta, \varphi)$.

The common assumptions on the bifunction $\Theta: C \times C \rightarrow \mathbf{R}$ to study GMEP (1.3) or EP (1.2) are the following:

(A1) $\Theta(x, x)=0$ for all $x \in C$;

(A2) $\Theta$ is monotone, i.e., $\Theta(x, y)+\Theta(y, x) \leq 0$ for any $x, y \in C$;

(A3) $\Theta$ is upper-hemicontinuous, i.e., for each $x, y, z \in C$,

$$
\limsup _{t \rightarrow 0^{+}} \Theta(t z+(1-t) x, y) \leq \Theta(x, y)
$$

(A4) $\Theta(x, \cdot)$ is convex and lower semicontinuous for each $x \in C$;

We use the assumption that the function $\varphi: C \rightarrow \mathbf{R}$ is a lower semicontinuous and convex function with restriction (B1) or (B2), where

(B1) for each $x \in H$ and $r>0$, there exists a bounded subset $D_{x} \subset C$ and $y_{x} \in C$ such that for any $z \in C \backslash D_{x}$,

$$
\Theta\left(z, y_{x}\right)+\varphi\left(y_{x}\right)-\varphi(z)+\frac{1}{r}\left\langle y_{x}-z, z-x\right\rangle<0 ;
$$

(B2) $C$ is a bounded set.

Given a positive number $r>0$. Let $T_{r}^{(\Theta, \varphi)}: H \rightarrow C$ be the solution set of the auxiliary mixed equilibrium problem, that is, for each $x \in H$,

$$
T_{r}^{(\Theta, \varphi)}(x):=\left\{y \in C: \Theta(y, z)+\varphi(z)-\varphi(y)+\frac{1}{r}\langle y-x, z-y\rangle \geq 0, \forall z \in C\right\} .
$$


Some elementary conclusions related to MEP are given in the following result.

Proposition 1.1 [11] Let $\Theta: C \times C \rightarrow \mathbf{R}$ satisfy conditions (A1)-(A4) and $\varphi: C \rightarrow \mathbf{R}$ be a proper lower semicontinuous and convex function such that either (B1) or (B2) holds. For $r>0$ and $x \in H$, define a mapping $T_{r}^{(\Theta, \varphi)}: H \rightarrow C$ by

$$
T_{r}^{(\Theta, \varphi)}(x)=\left\{z \in C: \Theta(z, y)+\varphi(y)-\varphi(z)+\frac{1}{r}\langle y-z, z-x\rangle \geq 0, \forall y \in C\right\}, \quad \forall x \in H .
$$

Then the following conclusions hold:

(i) For each $x \in H, T_{r}^{(\Theta, \varphi)}(x)$ is nonempty and single-valued;

(ii) $T_{r}^{(\Theta, \varphi)}$ is firmly nonexpansive, that is, for any $x, y \in H$,

$$
\left\|T_{r}^{(\Theta, \varphi)} x-T_{r}^{(\Theta, \varphi)} y\right\|^{2} \leq\left\langle T_{r}^{(\Theta, \varphi)} x-T_{r}^{(\Theta, \varphi)} y, x-y\right| ;
$$

(iii) $\operatorname{Fix}\left(T_{r}^{(\Theta, \varphi)}\right)=\operatorname{MEP}(\Theta, \varphi)$;

(iv) $\operatorname{MEP}(\Theta, \varphi)$ is closed and convex;

(v) $\left\|T_{s}^{(\Theta, \varphi)} x-T_{t}^{(\Theta, \varphi)} x\right\|^{2} \leq \frac{s-t}{s}\left\langle T_{s}^{(\Theta, \varphi)} x-T_{t}^{(\Theta, \varphi)} x, T_{s}^{(\Theta, \varphi)} x-x\right\rangle, \forall s, t>0$ and $x \in H$.

Recently, Cai and $\mathrm{Bu}$ [12] considered a problem of finding a common element of the set of solutions of finitely many generalized mixed equilibrium problems, the set of solutions of finitely many variational inequalities mappings and the set of fixed points of an asymptotically $k$-strict pseudocontractive mapping in the intermediate sense [13] in a real Hilbert space. They proposed and analyzed an algorithm for finding such a solution. The weak convergence result for the proposed algorithm is also presented.

\subsection{General system of variational inequalities}

Let $F_{1}, F_{2}: C \rightarrow H$ be two mappings. We consider the general system of variational inequalities (GSVI) of finding $\left(x^{*}, y^{*}\right) \in C \times C$ such that

$$
\begin{cases}\left\langle v_{1} F_{1} y^{*}+x^{*}-y^{*}, x-x^{*}\right\rangle \geq 0, & \forall x \in C, \\ \left\langle v_{2} F_{2} x^{*}+y^{*}-x^{*}, x-y^{*}\right\rangle \geq 0, & \forall x \in C,\end{cases}
$$

where $v_{1}>0$ and $v_{2}>0$ are two constants. It was considered and studied in $[5,14,15]$. In particular, if $F_{1}=F_{2}=A$, then the GSVI (1.4) reduces to the problem of finding $\left(x^{*}, y^{*}\right) \in$ $C \times C$ such that

$$
\begin{cases}\left\langle v_{1} A y^{*}+x^{*}-y^{*}, x-x^{*}\right\rangle \geq 0, \quad \forall x \in C, \\ \left\langle v_{2} A x^{*}+y^{*}-x^{*}, x-y^{*}\right\rangle \geq 0, \quad \forall x \in C .\end{cases}
$$

It is called a new system of variational inequalities (NSVI) [9]. It is worth to mention that the above system of two variational inequalities could be used to solve Nash equilibrium problem. For applications of system of variational inequalities to Nash equilibrium problems, we refer to [16-19] and the references therein. Further, if $x^{*}=y^{*}$ additionally, then the NSVI reduces to the classical VIP (1.1). Putting $G:=P_{C}\left(I-v_{1} F_{1}\right) P_{C}\left(I-v_{2} F_{2}\right)$ and $y^{*}=P_{C}\left(I-v_{2} F_{2}\right) x^{*}$, Ceng et al. [15] transformed the GSVI (1.4) into the following fixed point equation:

$$
G x^{*}=x^{*} .
$$




\subsection{Hierarchical variational inequalities}

A variational inequality problem defined over the set of fixed points of a nonexpansive mapping, is called a hierarchical variational inequality problem. Let $S, T: H \rightarrow H$ be nonexpansive mappings. We denote by $\operatorname{Fix}(T)$ and $\operatorname{Fix}(T)$ the set of fixed points of $T$ and $S$, respectively. If we replace $C$ by $\operatorname{Fix}(T)$ in the formulation of VIP (1.1), then VIP (1.1) is defined by $\operatorname{Fix}(T)$ and $A$ and it is called a hierarchical variational inequality problem.

Yao et al. [20] considered the hierarchical variational inequality problem (HVIP) in which the mapping $A$ is replaced by the monotone mapping $I-S$. They considered the following form of HVIP: find $\tilde{x} \in \operatorname{Fix}(T)$ such that

$$
\langle(I-S) \tilde{x}, p-\tilde{x}\rangle \geq 0, \quad \forall p \in \operatorname{Fix}(T) .
$$

The solution set of HVIP (1.7) is denoted by $\Lambda$. It is not hard to check that solving HVIP (1.7) is equivalent to the fixed point problem of the composite mapping $P_{\mathrm{Fix}(T)} \circ S$, that is, find $\tilde{x} \in C$ such that $\tilde{x}=P_{\operatorname{Fix}(T)} S \tilde{x}$. They proposed and analyzed an iterative method for solving this kind of HTVI. For further details and a comprehensive survey on HVIP, we refer to [21] and the references therein.

\subsection{Variational inclusions}

Let $B: C \rightarrow H$ be a single-valued mapping and $R: C \rightarrow 2^{H}$ be a set-valued mapping with $D(R)=C$. We consider the variational inclusion problem of finding a point $x \in C$ such that

$$
0 \in B x+R x
$$

We denote by $\mathrm{I}(B, R)$ the solution set of the variational inclusion (1.8). In particular, if $B \equiv R \equiv 0$, then $\mathrm{I}(B, R)=C$. If $B=0$, then problem (1.8) becomes the inclusion problem introduced by Rockafellar [22]. It is well known that problem (1.8) provides a convenient framework for the unified study of optimal solutions in many optimization related areas including mathematical programming, complementarity problems, variational inequalities, optimal control, mathematical economics, equilibria and game theory, etc.

\subsection{Problem to be considered}

In this paper, we introduce and study the following hierarchical variational inequality problem (HVIP) defined over a common set of solutions of finitely many GMEPs, finitely many variational inclusions, a general system of variational inequalities, and a fixed point of a strictly pseudocontractive mapping. Throughout the paper, we denote by $M$ and $N$ set of the positive integers.

Problem 1.1 Assume that

(i) for $j=1,2, F_{j}: C \rightarrow H$ and $F: H \rightarrow H$ are mappings;

(ii) for each $k \in\{1,2, \ldots, M\}, \Theta_{k}: C \times C \rightarrow \mathbf{R}$ is a bifunction satisfying conditions (A1)-(A4) and $\varphi_{k}: C \rightarrow \mathbf{R} \cup\{+\infty\}$ is a proper lower semicontinuous and convex function with restriction (B1) or (B2);

(iii) for each $k \in\{1,2, \ldots, M\}$ and $i \in\{1,2, \ldots, N\}, R_{i}: C \rightarrow 2^{H}$ is a maximal monotone mapping, and $A_{k}: H \rightarrow H$ and $B_{i}: C \rightarrow H$ are mappings;

(iv) $T: C \rightarrow C$ is a mapping and $S: H \rightarrow H$ is a nonexpansive mapping;

(v) $\Omega:=\bigcap_{k=1}^{M} \operatorname{GMEP}\left(\Theta_{k}, \varphi_{k}, A_{k}\right) \cap \bigcap_{i=1}^{N} \mathrm{I}\left(B_{i}, R_{i}\right) \cap \operatorname{GSVI}(G) \cap \operatorname{Fix}(T) \neq \emptyset$. 
Then the objective is to find $x^{*} \in \Omega$ such that

$$
\left\langle(\mu F-\gamma S) x^{*}, x-x^{*}\right\rangle \geq 0, \quad \forall x \in \Omega .
$$

By combining Korpelevich's extragradient method, the viscosity approximation method, the hybrid steepest-descent method [10], and Mann's iteration method, we introduce and analyze a multistep hybrid extragradient algorithm for finding a solution of Problem 1.1. It is proven that under appropriate assumptions, the proposed algorithm converges strongly to a solution of GSVI (1.4) defined over a common set of solutions of finitely many generalized mixed equilibrium problems (GMEPs), finitely many variational inclusions and the fixed point problem of a strictly pseudocontractive mapping. In the meantime, we also prove the strong convergence of the proposed algorithm to a unique solution of Problem 1.1. The results obtained in this paper improve and extend the corresponding results announced by many others.

\section{Preliminaries}

Throughout this paper, we assume that $H$ is a real Hilbert space whose inner product and norm are denoted by $\langle\cdot, \cdot\rangle$ and $\|\cdot\|$, respectively. Let $C$ be a nonempty closed convex subset of $H$. We write $x_{n} \rightarrow x$ to indicate that the sequence $\left\{x_{n}\right\}$ converges weakly to $x$ and $x_{n} \rightarrow x$ to indicate that the sequence $\left\{x_{n}\right\}$ converges strongly to $x$. Moreover, we use $\omega_{w}\left(x_{n}\right)$ to denote the weak $\omega$-limit set of the sequence $\left\{x_{n}\right\}$, i.e.,

$$
\omega_{w}\left(x_{n}\right):=\left\{x \in H: x_{n_{i}} \rightarrow x \text { for some subsequence }\left\{x_{n_{i}}\right\} \text { of }\left\{x_{n}\right\}\right\} .
$$

Definition 2.1 A mapping $A: C \rightarrow H$ is called

(i) $\eta$-strongly monotone if there exists a constant $\eta>0$ such that

$$
\langle A x-A y, x-y\rangle \geq \eta\|x-y\|^{2}, \quad \forall x, y \in C ;
$$

(ii) $\zeta$-inverse-strongly monotone if there exists a constant $\zeta>0$ such that

$$
\langle A x-A y, x-y\rangle \geq \zeta\|A x-A y\|^{2}, \quad \forall x, y \in C .
$$

It is easy to see that the projection $P_{C}$ is 1-inverse-strongly monotone. Inverse-strongly monotone (also referred to as co-coercive) operators have been applied widely in solving practical problems in various fields. It is obvious that if $A$ is $\zeta$-inverse-strongly monotone, then $A$ is monotone and $\frac{1}{\zeta}$-Lipschitz continuous. Moreover, we also have, for all $u, v \in C$ and $\lambda>0$,

$$
\begin{aligned}
\|(I-\lambda A) u-(I-\lambda A) v\|^{2} & =\|(u-v)-\lambda(A u-A v)\|^{2} \\
& =\|u-v\|^{2}-2 \lambda\langle A u-A v, u-v\rangle+\lambda^{2}\|A u-A v\|^{2} \\
& \leq\|u-v\|^{2}+\lambda(\lambda-2 \zeta)\|A u-A v\|^{2} .
\end{aligned}
$$

So, if $\lambda \leq 2 \zeta$, then $I-\lambda A$ is a nonexpansive mapping from $C$ to $H$. 
Definition 2.2 A mapping $T: H \rightarrow H$ is said to be firmly nonexpansive if $2 T-I$ is nonexpansive, or equivalently, if $T$ is 1-inverse-strongly monotone (1-ism),

$$
\langle x-y, T x-T y\rangle \geq\|T x-T y\|^{2}, \quad \forall x, y \in H
$$

alternatively, $T$ is firmly nonexpansive if and only if $T$ can be expressed as

$$
T=\frac{1}{2}(I+S)
$$

where $S: H \rightarrow H$ is nonexpansive; projections are firmly nonexpansive.

It can easily be seen that if $T$ is nonexpansive, then $I-T$ is monotone.

It is clear that, in a real Hilbert space $H, T: C \rightarrow C$ is $\xi$-strictly pseudocontractive if and only if the following inequality holds:

$$
\langle T x-T y, x-y\rangle \leq\|x-y\|^{2}-\frac{1-\xi}{2}\|(I-T) x-(I-T) y\|^{2}, \quad \forall x, y \in C .
$$

This immediately implies that if $T$ is a $\xi$-strictly pseudocontractive mapping, then $I-T$ is $\frac{1-\xi}{2}$-inverse-strongly monotone; for further details, we refer to [23] and the references therein. It is well known that the class of strict pseudocontractions strictly includes the class of nonexpansive mappings and that the class of pseudocontractions strictly includes the class of strict pseudocontractions.

Lemma 2.1 [23, Proposition 2.1] Let C be a nonempty closed convex subset of a real Hilbert space $H$ and $T: C \rightarrow C$ be a mapping.

(i) If $T$ is a $\xi$-strictly pseudocontractive mapping, then $T$ satisfies the Lipschitzian condition

$$
\|T x-T y\| \leq \frac{1+\xi}{1-\xi}\|x-y\|, \quad \forall x, y \in C .
$$

(ii) If $T$ is a $\xi$-strictly pseudocontractive mapping, then the mapping $I-T$ is semiclosed at 0 , that is, if $\left\{x_{n}\right\}$ is a sequence in $C$ such that $x_{n}-\tilde{x}$ and $(I-T) x_{n} \rightarrow 0$, then $(I-T) \tilde{x}=0$.

(iii) If $T$ is a $\xi$-(quasi-)strict pseudocontraction, then the fixed-point set $\operatorname{Fix}(T)$ of $T$ is closed and convex so that the projection $P_{\mathrm{Fix}(T)}$ is well defined.

Lemma 2.2 [24] Let $C$ be a nonempty closed convex subset of a real Hilbert space H. Let $T: C \rightarrow C$ be a $\xi$-strictly pseudocontractive mapping. Let $\gamma$ and $\delta$ be two nonnegative real numbers such that $(\gamma+\delta) \xi \leq \gamma$. Then

$$
\|\gamma(x-y)+\delta(T x-T y)\| \leq(\gamma+\delta)\|x-y\|, \quad \forall x, y \in C .
$$

Let $C$ be a nonempty closed convex subset of a real Hilbert space $H$. We introduce some notations. Let $\lambda$ be a number in $(0,1]$ and let $\mu>0$. Associated with a nonexpansive mapping $T: C \rightarrow H$, we define the mapping $T^{\lambda}: C \rightarrow H$ by

$$
T^{\lambda} x:=T x-\lambda \mu F(T x), \quad \forall x \in C,
$$


where $F: H \rightarrow H$ is an operator such that, for some positive constants $\kappa, \eta>0, F$ is $\kappa$ Lipschitzian and $\eta$-strongly monotone on $H$, that is, $F$ satisfies the conditions

$$
\|F x-F y\| \leq \kappa\|x-y\| \quad \text { and } \quad\langle F x-F y, x-y\rangle \geq \eta\|x-y\|^{2}
$$

for all $x, y \in H$.

Remark 2.1 Since $F$ is $\kappa$-Lipschitzian and $\eta$-strongly monotone on $H$, we get $0<\eta \leq \kappa$. Hence, whenever $0<\mu<\frac{2 \eta}{\kappa^{2}}$, we have

$$
\begin{aligned}
0 & \leq(1-\mu \eta)^{2}=1-2 \mu \eta+\mu^{2} \eta^{2} \\
& \leq 1-2 \mu \eta+\mu^{2} \kappa^{2}<1-2 \mu \eta+\frac{2 \eta}{\kappa^{2}} \mu \kappa^{2}=1,
\end{aligned}
$$

which implies

$$
0<1-\sqrt{1-2 \mu \eta+\mu^{2} \kappa^{2}} \leq 1 .
$$

So, $\tau=1-\sqrt{1-\mu\left(2 \eta-\mu \kappa^{2}\right)} \in(0,1]$.

Finally, recall that a set-valued mapping $T: D(T) \subset H \rightarrow 2^{H}$ is called monotone if for all $x, y \in D(T), f \in T x$ and $g \in T y$ imply

$$
\langle f-g, x-y\rangle \geq 0 .
$$

A set-valued mapping $T$ is called maximal monotone if $T$ is monotone and $(I+\lambda T) D(T)=$ $H$ for each $\lambda>0$, where $I$ is the identity mapping of $H$. We denote by $G(T)$ the graph of $T$. It is well known that a monotone mapping $T$ is maximal if and only if, for $(x, f) \in H \times H$, $\langle f-g, x-y\rangle \geq 0$ for every $(y, g) \in G(T)$ implies $f \in T x$. Next we provide an example to illustrate the concept of a maximal monotone mapping.

Let $A: C \rightarrow H$ be a monotone, $k$-Lipschitz-continuous mapping and let $N_{C} v$ be the normal cone to $C$ at $v \in C$, i.e.,

$$
N_{C} v=\{u \in H:\langle v-p, u\rangle \geq 0, \forall p \in C\} .
$$

Define

$$
\widetilde{T} v= \begin{cases}A v+N_{C} v, & \text { if } v \in C, \\ \emptyset, & \text { if } v \notin C .\end{cases}
$$

Then $\widetilde{T}$ is maximal monotone (see [22]) such that

$$
0 \in \widetilde{T} v \Longleftrightarrow v \in \operatorname{VI}(C, A) .
$$

Let $R: D(R) \subset H \rightarrow 2^{H}$ be a maximal monotone mapping. Let $\lambda, \mu>0$ be two positive numbers. Associated with $R$ and $\lambda$, we define the resolvent operator $J_{R, \lambda}: H \rightarrow \overline{D(R)}$ by

$$
J_{R, \lambda}=(I+\lambda R)^{-1}, \quad \forall x \in H,
$$

where $\lambda$ is a positive number. 
The lemma shows that the resolvent operator $J_{R, \lambda}: H \rightarrow \overline{D(R)}$ is nonexpansive.

Lemma 2.3 [25] $J_{R, \lambda}$ is single-valued and firmly nonexpansive, i.e.,

$$
\left\langle J_{R, \lambda} x-J_{R, \lambda} y, x-y\right\rangle \geq\left\|J_{R, \lambda} x-J_{R, \lambda} y\right\|^{2}, \quad \forall x, y \in H .
$$

Consequently, $J_{R, \lambda}$ is nonexpansive and monotone.

Lemma 2.4 [26] Let $R$ be a maximal monotone mapping with $D(R)=C$. Then for any given $\lambda>0, u \in C$ is a solution of problem (1.5) if and only if $u \in C$ satisfies

$$
u=J_{R, \lambda}(u-\lambda B u) .
$$

Lemma 2.5 [27] Let $R$ be a maximal monotone mapping with $D(R)=C$ and let $B: C \rightarrow H$ be a strongly monotone, continuous, and single-valued mapping. Then for each $z \in H$, the equation $z \in(B+\lambda R) x$ has a unique solution $x_{\lambda}$ for $\lambda>0$.

Lemma 2.6 [26] Let $R$ be a maximal monotone mapping with $D(R)=C$ and $B: C \rightarrow H$ be a monotone, continuous and single-valued mapping. Then $(I+\lambda(R+B)) C=H$ for each $\lambda>0$. In this case, $R+B$ is maximal monotone.

Lemma 2.7 [28] We have the resolvent identity

$$
J_{R, \lambda} x=J_{R, \mu}\left(\frac{\mu}{\lambda} x+\left(1-\frac{\mu}{\lambda}\right) J_{R, \lambda} x\right), \quad \forall x \in H
$$

Remark 2.2 For $\lambda, \mu>0$, we have the following relation:

$$
\left\|J_{R, \lambda} x-J_{R, \mu} y\right\| \leq\|x-y\|+|\lambda-\mu|\left(\frac{1}{\lambda}\left\|J_{R, \lambda} x-y\right\|+\frac{1}{\mu}\left\|x-J_{R, \mu} y\right\|\right), \quad \forall x, y \in H .
$$

Indeed, whenever $\lambda \geq \mu$, utilizing Lemma 2.7 we deduce that

$$
\begin{aligned}
\left\|J_{R, \lambda} x-J_{R, \mu} y\right\| & =\left\|J_{R, \mu}\left(\frac{\mu}{\lambda} x+\left(1-\frac{\mu}{\lambda}\right) J_{R, \lambda} x\right)-J_{R, \mu} y\right\| \\
& \leq\left\|\frac{\mu}{\lambda} x+\left(1-\frac{\mu}{\lambda}\right) J_{R, \lambda} x-y\right\| \\
& \leq \frac{\mu}{\lambda}\|x-y\|+\left(1-\frac{\mu}{\lambda}\right)\left\|J_{R, \lambda} x-y\right\| \\
& \leq\|x-y\|+\frac{|\lambda-\mu|}{\lambda}\left\|J_{R, \lambda} x-y\right\| .
\end{aligned}
$$

Similarly, whenever $\lambda<\mu$, we get

$$
\left\|J_{R, \lambda} x-J_{R, \mu} y\right\| \leq\|x-y\|+\frac{|\lambda-\mu|}{\mu}\left\|x-J_{R, \mu} y\right\| .
$$

Combining the above two cases we conclude that (2.3) holds. 
We need following fact and lemmas to establish the strong convergence of the sequences generated by the proposed algorithm.

Lemma 2.8 Let $X$ be a real inner product space. Then

$$
\|x+y\|^{2} \leq\|x\|^{2}+2\langle y, x+y\rangle, \quad \forall x, y \in X .
$$

Lemma 2.9 Let $H$ be a real Hilbert space. Then:

(a) $\|x-y\|^{2}=\|x\|^{2}-\|y\|^{2}-2\langle x-y, y\rangle$ for all $x, y \in H$;

(b) $\|\lambda x+\mu y\|^{2}=\lambda\|x\|^{2}+\mu\|y\|^{2}-\lambda \mu\|x-y\|^{2}$ for all $x, y \in H$ and $\lambda, \mu \in[0,1]$ with $\lambda+\mu=1$

(c) If $\left\{x_{n}\right\}$ is a sequence in $H$ such that $x_{n} \rightarrow x$, it follows that

$$
\limsup _{n \rightarrow \infty}\left\|x_{n}-y\right\|^{2}=\limsup _{n \rightarrow \infty}\left\|x_{n}-x\right\|^{2}+\|x-y\|^{2}, \quad \forall y \in H .
$$

Lemma 2.10 (Demiclosedness principle [29]) Let C be a nonempty closed convex subset of a real Hilbert space $H$. Let $S$ be a nonexpansive self-mapping on $C$ with $\operatorname{Fix}(S) \neq \emptyset$. Then $I-S$ is demiclosed, that is, whenever $\left\{x_{n}\right\}$ is a sequence in $C$ weakly converging to some $x \in C$ and the sequence $\left\{(I-S) x_{n}\right\}$ strongly converges to some $y$, it follows that $(I-S) x=y$, where $I$ is the identity operator of $H$.

Lemma 2.11 [30, Lemma 3.1] $T^{\lambda}$ is a contraction provided $0<\mu<\frac{2 \eta}{\kappa^{2}}$; that is,

$$
\left\|T^{\lambda} x-T^{\lambda} y\right\| \leq(1-\lambda \tau)\|x-y\|, \quad \forall x, y \in C,
$$

where $\tau=1-\sqrt{1-\mu\left(2 \eta-\mu \kappa^{2}\right)} \in(0,1]$.

Remark 2.3 (a) Since $F$ is $\kappa$-Lipschitzian and $\eta$-strongly monotone on $H$, we get $0<$ $\eta \leq \kappa$. Hence, whenever $0<\mu<\frac{2 \eta}{\kappa^{2}}$, we have

$$
\begin{aligned}
0 & \leq(1-\mu \eta)^{2}=1-2 \mu \eta+\mu^{2} \eta^{2} \\
& \leq 1-2 \mu \eta+\mu^{2} \kappa^{2} \\
& <1-2 \mu \eta+\frac{2 \eta}{\kappa^{2}} \mu \kappa^{2}=1,
\end{aligned}
$$

which implies

$$
0<1-\sqrt{1-2 \mu \eta+\mu^{2} \kappa^{2}} \leq 1 .
$$

Therefore, $\tau=1-\sqrt{1-\mu\left(2 \eta-\mu \kappa^{2}\right)} \in(0,1]$.

(b) In Lemma 2.11, put $F=\frac{1}{2} I$ and $\mu=2$. Then we know that $\kappa=\eta=\frac{1}{2}, 0<\mu=2<\frac{2 \eta}{\kappa^{2}}=4$ and

$$
\tau=1-\sqrt{1-\mu\left(2 \eta-\mu \kappa^{2}\right)}=1-\sqrt{1-2\left(2 \times \frac{1}{2}-2 \times\left(\frac{1}{2}\right)^{2}\right)}=1 .
$$


Lemma 2.12 [30] Let $\left\{s_{n}\right\}$ be a sequence of nonnegative numbers satisfying the conditions

$$
s_{n+1} \leq\left(1-\alpha_{n}\right) s_{n}+\alpha_{n} \beta_{n}, \quad \forall n \geq 1,
$$

where $\left\{\alpha_{n}\right\}$ and $\left\{\beta_{n}\right\}$ are sequences of real numbers such that

(a) $\left\{\alpha_{n}\right\} \subset[0,1]$ and $\sum_{n=1}^{\infty} \alpha_{n}=\infty$, or equivalently,

$$
\prod_{n=1}^{\infty}\left(1-\alpha_{n}\right):=\lim _{n \rightarrow \infty} \prod_{k=1}^{n}\left(1-\alpha_{k}\right)=0
$$

(b) $\lim \sup _{n \rightarrow \infty} \beta_{n} \leq 0$, or $\sum_{n=1}^{\infty}\left|\alpha_{n} \beta_{n}\right|<\infty$.

Then $\lim _{n \rightarrow \infty} s_{n}=0$.

\section{Algorithms and convergence results}

In this section, we will introduce and analyze a multistep hybrid extragradient algorithm for finding a solution of the HVIP (1.9) (over the fixed point set of a strictly pseudocontractive mapping) with constraints of several problems: GSVI (1.4), finitely many GMEPs, and finitely many variational inclusions in a real Hilbert space. This algorithm is based on Korpelevich's extragradient method, the viscosity approximation method, the hybrid steepest-descent method [10], and Mann's iteration method. We prove the strong convergence of the proposed algorithm to a unique solution of HVIP (1.9) under suitable conditions.

We propose the following algorithm to compute the approximate solution of Problem 1.1.

Algorithm 3.1 Let $C$ be a nonempty closed convex subset of a real Hilbert space $H$. For each $k \in\{1,2, \ldots, M\}$, let $\Theta_{k}: C \times C \rightarrow \mathbf{R}$ be a bifunction satisfying conditions (A1)-(A4) and $\varphi_{k}: C \rightarrow \mathbf{R} \cup\{+\infty\}$ be a proper lower semicontinuous and convex function with restriction (B1) or (B2). For each $k \in\{1,2, \ldots, M\}, i \in\{1,2, \ldots, N\}$, let $R_{i}: C \rightarrow 2^{H}$ be a maximal monotone mapping, and $A_{k}: H \rightarrow H$ and $B_{i}: C \rightarrow H$ be $\mu_{k}$-inverse-strongly monotone and $\eta_{i}$-inverse-strongly monotone, respectively. Let $T: C \rightarrow C$ be a $\xi$-strictly pseudocontractive mapping, $S: H \rightarrow H$ is a nonexpansive mapping and $V: H \rightarrow H$ be a $\rho$-contraction with coefficient $\rho \in[0,1)$. For each $j=1,2$, let $F_{j}: C \rightarrow H$ be $\zeta_{j}$-inversestrongly monotone and $F: H \rightarrow H$ be $\kappa$-Lipschitzian and $\eta$-strongly monotone with positive constants $\kappa, \eta>0$ such that $0 \leq \gamma<\tau$ and $0<\mu<\frac{2 \eta}{\kappa^{2}}$ where $\tau=1-\sqrt{1-\mu\left(2 \eta-\mu \kappa^{2}\right)}$. Assume that $\Omega:=\bigcap_{k=1}^{M} \operatorname{GMEP}\left(\Theta_{k}, \varphi_{k}, A_{k}\right) \cap \bigcap_{i=1}^{N} \mathrm{I}\left(B_{i}, R_{i}\right) \cap \operatorname{GSVI}(G) \cap \operatorname{Fix}(T) \neq \emptyset$. Let $\left\{\alpha_{n}\right\},\left\{\lambda_{n}\right\} \subset(0,1],\left\{\beta_{n}\right\},\left\{\gamma_{n}\right\},\left\{\delta_{n}\right\} \subset[0,1],\left\{\rho_{n}\right\} \subset(0,2 \alpha],\left\{\lambda_{i, n}\right\} \subset\left[a_{i}, b_{i}\right] \subset\left(0,2 \eta_{i}\right)$ and $\left\{r_{k, n}\right\} \subset\left[c_{k}, d_{k}\right] \subset\left(0,2 \mu_{k}\right)$ where $i \in\{1,2, \ldots, N\}$ and $k \in\{1,2, \ldots, M\}$. For arbitrarily given $x_{0} \in H$, let $\left\{x_{n}\right\}$ be a sequence generated by

$$
\left\{\begin{array}{l}
u_{n}=T_{r_{M, n}}^{\left(\Theta_{M}, \varphi_{M}\right)}\left(I-r_{M, n} A_{M}\right) T_{r_{M-1, n}}^{\left(\Theta_{M-1}, \varphi_{M-1}\right)}\left(I-r_{M-1, n} A_{M-1}\right) \cdots T_{r_{1, n}}^{\left(\Theta_{1}, \varphi_{1}\right)}\left(I-r_{1, n} A_{1}\right) x_{n}, \\
v_{n}=J_{R_{N}, \lambda_{N, n}}\left(I-\lambda_{N, n} B_{N}\right) J_{R_{N-1}, \lambda_{N-1, n}}\left(I-\lambda_{N-1, n} B_{N-1}\right) \cdots J_{R_{1}, \lambda_{1, n}}\left(I-\lambda_{1, n} B_{1}\right) u_{n}, \\
y_{n}=\beta_{n} x_{n}+\gamma_{n} G v_{n}+\delta_{n} T G v_{n}, \\
x_{n+1}=\lambda_{n} \gamma\left(\alpha_{n} V x_{n}+\left(1-\alpha_{n}\right) S x_{n}\right)+\left(I-\lambda_{n} \mu F\right) y_{n}, \quad \forall n \geq 0
\end{array}\right.
$$

where $G:=P_{C}\left(I-v_{1} F_{1}\right) P_{C}\left(I-v_{2} F_{2}\right)$ with $v_{j} \in\left(0,2 \zeta_{j}\right)$ for $j=1,2$. 
Theorem 3.1 In addition to the assumption in Algorithm 3.1, suppose that

(i) $\lim _{n \rightarrow \infty} \lambda_{n}=0, \sum_{n=0}^{\infty} \lambda_{n}=\infty$ and $\lim _{n \rightarrow \infty} \frac{1}{\lambda_{n}}\left|1-\frac{\alpha_{n-1}}{\alpha_{n}}\right|=0$;

(ii) $\lim \sup _{n \rightarrow \infty} \frac{\alpha_{n}}{\lambda_{n}}<\infty, \lim _{n \rightarrow \infty} \frac{1}{\lambda_{n}}\left|\frac{1}{\alpha_{n}}-\frac{1}{\alpha_{n-1}}\right|=0$ and $\lim _{n \rightarrow \infty} \frac{1}{\alpha_{n}}\left|1-\frac{\lambda_{n-1}}{\lambda_{n}}\right|=0$;

(iii) $\lim _{n \rightarrow \infty} \frac{\left|\beta_{n}-\beta_{n-1}\right|}{\lambda_{n} \alpha_{n}}=0$ and $\lim _{n \rightarrow \infty} \frac{\left|\gamma_{n}-\gamma_{n-1}\right|}{\lambda_{n} \alpha_{n}}=0$;

(iv) $\lim _{n \rightarrow \infty} \frac{\left|\lambda_{i, n}-\lambda_{i, n-1}\right|}{\lambda_{n} \alpha_{n}}=0$ and $\lim _{n \rightarrow \infty} \frac{\left|r_{k, n}-r_{k, n-1}\right|}{\lambda_{n} \alpha_{n}}=0$ for $i=1,2, \ldots, N$ and

$$
k=1,2, \ldots, M
$$

(v) $\beta_{n}+\gamma_{n}+\delta_{n}=1$ and $\left(\gamma_{n}+\delta_{n}\right) \xi \leq \gamma_{n}$ for all $n \geq 0$;

(vi) $\left\{\beta_{n}\right\} \subset[a, b] \subset(0,1)$ and $\liminf _{n \rightarrow \infty} \delta_{n}>0$.

Then

(a) $\lim _{n \rightarrow \infty} \frac{\left\|x_{n+1}-x_{n}\right\|}{\alpha_{n}}=0$

(b) $\omega_{w}\left(x_{n}\right) \subset \Omega$;

(c) $\left\{x_{n}\right\}$ converges strongly to a point $x^{*} \in \Omega$, which is a unique solution of HVIP (1.9), that is,

$$
\left\langle(\mu F-\gamma S) x^{*}, p-x^{*}\right\rangle \geq 0, \quad \forall p \in \Omega .
$$

Proof First of all, observe that

$$
\begin{aligned}
\mu \eta \geq \tau & \Longleftrightarrow \mu \eta \geq 1-\sqrt{1-\mu\left(2 \eta-\mu \kappa^{2}\right)} \\
& \Longleftrightarrow \sqrt{1-\mu\left(2 \eta-\mu \kappa^{2}\right)} \geq 1-\mu \eta \\
& \Longleftrightarrow 1-2 \mu \eta+\mu^{2} \kappa^{2} \geq 1-2 \mu \eta+\mu^{2} \eta^{2} \\
& \Longleftrightarrow \kappa^{2} \geq \eta^{2} \\
& \Longleftrightarrow \kappa \geq \eta
\end{aligned}
$$

and

$$
\begin{aligned}
\langle(\mu F-\gamma S) x-(\mu F-\gamma S) y, x-y\rangle & =\mu\langle F x-F y, x-y\rangle-\gamma\langle S x-S y, x-y\rangle \\
& \geq \mu \eta\|x-y\|^{2}-\gamma\|x-y\|^{2} \\
& =(\mu \eta-\gamma)\|x-y\|^{2}, \quad \forall x, y \in H .
\end{aligned}
$$

Since $0 \leq \gamma<\tau$ and $\kappa \geq \eta$, we know that $\mu \eta \geq \tau>\gamma$ and hence the mapping $\mu F-\gamma S$ is $(\mu \eta-\gamma)$-strongly monotone. Moreover, it is clear that the mapping $\mu F-\gamma S$ is $(\mu \kappa+\gamma)$ Lipschitzian. Thus, there exists a unique solution $x^{*}$ in $\Omega$ to the VIP

$$
\left\langle(\mu F-\gamma S) x^{*}, p-x^{*}\right\rangle \geq 0, \quad \forall p \in \Omega .
$$

That is, $\left\{x^{*}\right\}=\operatorname{VI}(\Omega, \mu F-\gamma S)$. Now, we put

$$
\Delta_{n}^{k}=T_{r_{k, n}}^{\left(\Theta_{k}, \varphi_{k}\right)}\left(I-r_{k, n} A_{k}\right) T_{r_{k-1, n}}^{\left(\Theta_{k-1}, \varphi_{k-1}\right)}\left(I-r_{k-1, n} A_{k-1}\right) \cdots T_{r_{1, n}}^{\left(\Theta_{1}, \varphi_{1}\right)}\left(I-r_{1, n} A_{1}\right) x_{n}
$$

for all $k \in\{1,2, \ldots, M\}$ and $n \geq 1$,

$$
\Lambda_{n}^{i}=J_{R_{i}, \lambda_{i, n}}\left(I-\lambda_{i, n} B_{i}\right) J_{R_{i-1}, \lambda_{i-1, n}}\left(I-\lambda_{i-1, n} B_{i-1}\right) \cdots J_{R_{1}, \lambda_{1, n}}\left(I-\lambda_{1, n} B_{1}\right)
$$


for all $i \in\{1,2, \ldots, N\}, \Delta_{n}^{0}=I$ and $\Lambda_{n}^{0}=I$, where $I$ is the identity mapping on $H$. Then we have $u_{n}=\Delta_{n}^{M} x_{n}$ and $v_{n}=\Lambda_{n}^{N} u_{n}$.

We divide the rest of the proof into several steps.

Step 1 . We prove that $\left\{x_{n}\right\}$ is bounded.

Indeed, take a fixed $p \in \Omega$ arbitrarily. Utilizing (2.1) and Proposition 1.1(ii), we have

$$
\begin{aligned}
& \left\|u_{n}-p\right\|=\left\|T_{r_{M, n}}^{\left(\Theta_{M}, \varphi_{M}\right)}\left(I-r_{M, n} B_{M}\right) \Delta_{n}^{M-1} x_{n}-T_{r_{M, n}}^{\left(\Theta_{M}, \varphi_{M}\right)}\left(I-r_{M, n} B_{M}\right) \Delta_{n}^{M-1} p\right\| \\
& \leq\left\|\left(I-r_{M, n} B_{M}\right) \Delta_{n}^{M-1} x_{n}-\left(I-r_{M, n} B_{M}\right) \Delta_{n}^{M-1} p\right\| \\
& \leq\left\|\Delta_{n}^{M-1} x_{n}-\Delta_{n}^{M-1} p\right\| \\
& \leq\left\|\Delta_{n}^{0} x_{n}-\Delta_{n}^{0} p\right\| \\
& =\left\|x_{n}-p\right\| \text {. }
\end{aligned}
$$

Utilizing (2.1) and Lemma 2.3, we have

$$
\begin{aligned}
\left\|v_{n}-p\right\|= & \left\|J_{R_{N}, \lambda_{N, n}}\left(I-\lambda_{N, n} A_{N}\right) \Lambda_{n}^{N-1} u_{n}-J_{R_{N}, \lambda_{N, n}}\left(I-\lambda_{N, n} A_{N}\right) \Lambda_{n}^{N-1} p\right\| \\
\leq & \left\|\left(I-\lambda_{N, n} A_{N}\right) \Lambda_{n}^{N-1} u_{n}-\left(I-\lambda_{N, n} A_{N}\right) \Lambda_{n}^{N-1} p\right\| \\
\leq & \left\|\Lambda_{n}^{N-1} u_{n}-\Lambda_{n}^{N-1} p\right\| \\
& \cdots \\
\leq & \left\|\Lambda_{n}^{0} u_{n}-\Lambda_{n}^{0} p\right\| \\
= & \left\|u_{n}-p\right\| .
\end{aligned}
$$

Combining (3.2) and (3.3), we have

$$
\left\|v_{n}-p\right\| \leq\left\|x_{n}-p\right\| .
$$

Since $p=G p=P_{C}\left(I-v_{1} F_{1}\right) P_{C}\left(I-v_{2} F_{2}\right) p, F_{j}$ is $\zeta_{j}$-inverse-strongly monotone for $j=1,2$, and $0<v_{j} \leq 2 \zeta_{j}$ for $j=1,2$, we deduce that, for any $n \geq 0$,

$$
\begin{aligned}
&\left\|G v_{n}-p\right\|^{2} \\
&=\left\|P_{C}\left(I-v_{1} F_{1}\right) P_{C}\left(I-v_{2} F_{2}\right) v_{n}-P_{C}\left(I-v_{1} F_{1}\right) P_{C}\left(I-v_{2} F_{2}\right) p\right\|^{2} \\
& \leq\left\|\left(I-v_{1} F_{1}\right) P_{C}\left(I-v_{2} F_{2}\right) v_{n}-\left(I-v_{1} F_{1}\right) P_{C}\left(I-v_{2} F_{2}\right) p\right\|^{2} \\
&=\left\|\left[P_{C}\left(I-v_{2} F_{2}\right) v_{n}-P_{C}\left(I-v_{2} F_{2}\right) p\right]-v_{1}\left[F_{1} P_{C}\left(I-v_{2} F_{2}\right) v_{n}-F_{1} P_{C}\left(I-v_{2} F_{2}\right) p\right]\right\|^{2} \\
& \leq\left\|P_{C}\left(I-v_{2} F_{2}\right) v_{n}-P_{C}\left(I-v_{2} F_{2}\right) p\right\|^{2} \\
&+v_{1}\left(v_{1}-2 \zeta_{1}\right)\left\|F_{1} P_{C}\left(I-v_{2} F_{2}\right) v_{n}-F_{1} P_{C}\left(I-v_{2} F_{2}\right) p\right\|^{2} \\
& \leq\left\|P_{C}\left(I-v_{2} F_{2}\right) v_{n}-P_{C}\left(I-v_{2} F_{2}\right) p\right\|^{2} \\
& \leq\left\|\left(I-v_{2} F_{2}\right) v_{n}-\left(I-v_{2} F_{2}\right) p\right\|^{2} \\
&=\left\|\left(v_{n}-p\right)-v_{2}\left(F_{2} v_{n}-F_{2} p\right)\right\|^{2}
\end{aligned}
$$




$$
\begin{aligned}
& \leq\left\|v_{n}-p\right\|^{2}+v_{2}\left(v_{2}-2 \zeta_{2}\right)\left\|F_{2} v_{n}-F_{2} p\right\|^{2} \\
& \leq\left\|v_{n}-p\right\|^{2} .
\end{aligned}
$$

(This shows that $G: C \rightarrow C$ is a nonexpansive mapping.) Since $\left(\gamma_{n}+\delta_{n}\right) \xi \leq \gamma_{n}$ for all $n \geq 0$ and $T$ is $\xi$-strictly pseudocontractive, utilizing Lemma 2.2, we obtain from (3.1), (3.4), and (3.5)

$$
\begin{aligned}
\left\|y_{n}-p\right\| & =\left\|\beta_{n} x_{n}+\gamma_{n} G v_{n}+\delta_{n} T G v_{n}-p\right\| \\
& =\left\|\beta_{n}\left(x_{n}-p\right)+\gamma_{n}\left(G v_{n}-p\right)+\delta_{n}\left(T G v_{n}-p\right)\right\| \\
& \leq \beta_{n}\left\|x_{n}-p\right\|+\left\|\gamma_{n}\left(G v_{n}-p\right)+\delta_{n}\left(T G v_{n}-p\right)\right\| \\
& \leq \beta_{n}\left\|x_{n}-p\right\|+\left(\gamma_{n}+\delta_{n}\right)\left\|G v_{n}-p\right\| \\
& \leq \beta_{n}\left\|x_{n}-p\right\|+\left(\gamma_{n}+\delta_{n}\right)\left\|v_{n}-p\right\| \\
& \leq \beta_{n}\left\|x_{n}-p\right\|+\left(\gamma_{n}+\delta_{n}\right)\left\|x_{n}-p\right\| \\
& =\left\|x_{n}-p\right\| .
\end{aligned}
$$

Utilizing Lemma 2.11, we deduce from (3.1), (3.6), and $0 \leq \gamma<\tau$ that for all $n \geq 0$

$$
\begin{aligned}
\left\|x_{n+1}-p\right\| & \\
= & \left\|\lambda_{n} \gamma\left(\alpha_{n} V x_{n}+\left(1-\alpha_{n}\right) S x_{n}\right)+\left(I-\lambda_{n} \mu F\right) y_{n}-p\right\| \\
= & \left\|\lambda_{n} \gamma\left(\alpha_{n} V x_{n}+\left(1-\alpha_{n}\right) S x_{n}\right)-\lambda_{n} \mu F p+\left(I-\lambda_{n} \mu F\right) y_{n}-\left(I-\lambda_{n} \mu F\right) p\right\| \\
\leq & \left\|\lambda_{n} \gamma\left(\alpha_{n} V x_{n}+\left(1-\alpha_{n}\right) S x_{n}\right)-\lambda_{n} \mu F p\right\|+\left\|\left(I-\lambda_{n} \mu F\right) y_{n}-\left(I-\lambda_{n} \mu F\right) p\right\| \\
= & \lambda_{n}\left\|\alpha_{n}\left(\gamma V x_{n}-\mu F p\right)+\left(1-\alpha_{n}\right)\left(\gamma S x_{n}-\mu F p\right)\right\|+\left\|\left(I-\lambda_{n} \mu F\right) y_{n}-\left(I-\lambda_{n} \mu F\right) p\right\| \\
\leq & \lambda_{n}\left[\alpha_{n}\left\|\gamma V x_{n}-\mu F p\right\|+\left(1-\alpha_{n}\right)\left\|\gamma S x_{n}-\mu F p\right\|\right]+\left\|\left(I-\lambda_{n} \mu F\right) y_{n}-\left(I-\lambda_{n} \mu F\right) p\right\| \\
\leq & \lambda_{n}\left[\alpha_{n}\left(\gamma\left\|V x_{n}-V p\right\|+\|\gamma V p-\mu F p\|\right)+\left(1-\alpha_{n}\right)\left(\gamma\left\|S x_{n}-S p\right\|+\|\gamma S p-\mu F p\|\right)\right] \\
& +\left\|\left(I-\lambda_{n} \mu F\right) y_{n}-\left(I-\lambda_{n} \mu F\right) p\right\| \\
\leq & \lambda_{n}\left[\alpha_{n}\left(\gamma \rho\left\|x_{n}-p\right\|+\|\gamma V p-\mu F p\|\right)+\left(1-\alpha_{n}\right)\left(\gamma\left\|x_{n}-p\right\|+\|\gamma S p-\mu F p\|\right)\right] \\
& +\left(1-\lambda_{n} \tau\right)\left\|y_{n}-p\right\| \\
\leq & \lambda_{n}\left[\left(1-\alpha_{n}(1-\rho)\right) \gamma\left\|x_{n}-p\right\|+\max \{\|\gamma V p-\mu F p\|,\|\gamma S p-\mu F p\|\}\right] \\
& +\left(1-\lambda_{n} \tau\right)\left\|x_{n}-p\right\| \\
= & \lambda_{n}\left(1-\alpha_{n}(1-\rho)\right) \gamma\left\|x_{n}-p\right\|+\lambda_{n} \max \{\|\gamma V p-\mu F p\|,\|\gamma S p-\mu F p\|\} \\
& +\left(1-\lambda_{n} \tau\right)\left\|x_{n}-p\right\| \\
\leq & \lambda_{n} \gamma\left\|x_{n}-p\right\|+\lambda_{n} \max \{\|\gamma V p-\mu F p\|,\|\gamma S p-\mu F p\|\}+\left(1-\lambda_{n} \tau\right)\left\|x_{n}-p\right\| \\
= & \left(1-\lambda_{n}(\tau-\gamma)\right)\left\|x_{n}-p\right\|+\lambda_{n} \max \{\|\gamma V p-\mu F p\|,\|\gamma S p-\mu F p\|\} \\
= & \left(1-\lambda_{n}(\tau-\gamma)\right)\left\|x_{n}-p\right\|+\lambda_{n}(\tau-\gamma) \frac{\max \{\|\gamma V p-\mu F p\|,\|\gamma S p-\mu F p\|\}}{\tau-\gamma} \\
\leq & \max \left\{\left\|x_{n}-p\right\|, \frac{\|\gamma V p-\mu F p\|}{\tau-\gamma}, \frac{\|\gamma S p-\mu F p\|}{\tau-\gamma}\right\} . \\
&
\end{aligned}
$$


By induction, we get

$$
\left\|x_{n}-p\right\| \leq \max \left\{\left\|x_{0}-p\right\|, \frac{\|\gamma V p-\mu F p\|}{\tau-\gamma}, \frac{\|\gamma S p-\mu F p\|}{\tau-\gamma}\right\}, \quad \forall n \geq 0
$$

Thus, $\left\{x_{n}\right\}$ is bounded and so are the sequences $\left\{u_{n}\right\},\left\{v_{n}\right\}$, and $\left\{y_{n}\right\}$.

Step 2. We prove that $\lim _{n \rightarrow \infty} \frac{\left\|x_{n+1}-x_{n}\right\|}{\alpha_{n}}=0$.

Indeed, utilizing (2.1) and (2.3), we obtain

$$
\begin{aligned}
& \left\|v_{n+1}-v_{n}\right\| \\
& =\left\|\Lambda_{n+1}^{N} u_{n+1}-\Lambda_{n}^{N} u_{n}\right\| \\
& =\left\|J_{R_{N}, \lambda_{N, n+1}}\left(I-\lambda_{N, n+1} B_{N}\right) \Lambda_{n+1}^{N-1} u_{n+1}-J_{R_{N}, \lambda_{N, n}}\left(I-\lambda_{N, n} B_{N}\right) \Lambda_{n}^{N-1} u_{n}\right\| \\
& \leq\left\|J_{R_{N}, \lambda_{N, n+1}}\left(I-\lambda_{N, n+1} B_{N}\right) \Lambda_{n+1}^{N-1} u_{n+1}-J_{R_{N}, \lambda_{N, n+1}}\left(I-\lambda_{N, n} B_{N}\right) \Lambda_{n+1}^{N-1} u_{n+1}\right\| \\
& +\left\|J_{R_{N}, \lambda_{N, n+1}}\left(I-\lambda_{N, n} B_{N}\right) \Lambda_{n+1}^{N-1} u_{n+1}-J_{R_{N}, \lambda_{N, n}}\left(I-\lambda_{N, n} B_{N}\right) \Lambda_{n}^{N-1} u_{n}\right\| \\
& \leq\left\|\left(I-\lambda_{N, n+1} B_{N}\right) \Lambda_{n+1}^{N-1} u_{n+1}-\left(I-\lambda_{N, n} B_{N}\right) \Lambda_{n+1}^{N-1} u_{n+1}\right\| \\
& +\left\|\left(I-\lambda_{N, n} B_{N}\right) \Lambda_{n+1}^{N-1} u_{n+1}-\left(I-\lambda_{N, n} B_{N}\right) \Lambda_{n}^{N-1} u_{n}\right\|+\left|\lambda_{N, n+1}-\lambda_{N, n}\right| \\
& \times\left(\frac{1}{\lambda_{N, n+1}}\left\|J_{R_{N}, \lambda_{N, n+1}}\left(I-\lambda_{N, n} B_{N}\right) \Lambda_{n+1}^{N-1} u_{n+1}-\left(I-\lambda_{N, n} B_{N}\right) \Lambda_{n}^{N-1} u_{n}\right\|\right. \\
& \left.+\frac{1}{\lambda_{N, n}}\left\|\left(I-\lambda_{N, n} B_{N}\right) \Lambda_{n+1}^{N-1} u_{n+1}-J_{R_{N}, \lambda_{N, n}}\left(I-\lambda_{N, n} B_{N}\right) \Lambda_{n}^{N-1} u_{n}\right\|\right) \\
& \leq\left|\lambda_{N, n+1}-\lambda_{N, n}\right|\left(\left\|B_{N} \Lambda_{n+1}^{N-1} u_{n+1}\right\|+\tilde{M}\right)+\left\|\Lambda_{n+1}^{N-1} u_{n+1}-\Lambda_{n}^{N-1} u_{n}\right\| \\
& \leq\left|\lambda_{N, n+1}-\lambda_{N, n}\right|\left(\left\|B_{N} \Lambda_{n+1}^{N-1} u_{n+1}\right\|+\tilde{M}\right) \\
& +\left|\lambda_{N-1, n+1}-\lambda_{N-1, n}\right|\left(\left\|B_{N-1} \Lambda_{n+1}^{N-2} u_{n+1}\right\|+\tilde{M}\right)+\left\|\Lambda_{n+1}^{N-2} u_{n+1}-\Lambda_{n}^{N-2} u_{n}\right\| \\
& \text {.. } \\
& \leq\left|\lambda_{N, n+1}-\lambda_{N, n}\right|\left(\left\|B_{N} \Lambda_{n+1}^{N-1} u_{n+1}\right\|+\tilde{M}\right)+\left|\lambda_{N-1, n+1}-\lambda_{N-1, n}\right|\left(\left\|B_{N-1} \Lambda_{n+1}^{N-2} u_{n+1}\right\|+\tilde{M}\right) \\
& +\cdots+\left|\lambda_{1, n+1}-\lambda_{1, n}\right|\left(\left\|B_{1} \Lambda_{n+1}^{0} u_{n+1}\right\|+\tilde{M}\right)+\left\|\Lambda_{n+1}^{0} u_{n+1}-\Lambda_{n}^{0} u_{n}\right\| \\
& \leq \tilde{M}_{0} \sum_{i=1}^{N}\left|\lambda_{i, n+1}-\lambda_{i, n}\right|+\left\|u_{n+1}-u_{n}\right\|
\end{aligned}
$$

where

$$
\begin{aligned}
& \sup _{n \geq 0}\left\{\frac{1}{\lambda_{N, n+1}}\left\|J_{R_{N}, \lambda_{N, n+1}}\left(I-\lambda_{N, n} B_{N}\right) \Lambda_{n+1}^{N-1} u_{n+1}-\left(I-\lambda_{N, n} B_{N}\right) \Lambda_{n}^{N-1} u_{n}\right\|\right. \\
& \left.\quad+\frac{1}{\lambda_{N, n}}\left\|\left(I-\lambda_{N, n} B_{N}\right) \Lambda_{n+1}^{N-1} u_{n+1}-J_{R_{N}, \lambda_{N, n}}\left(I-\lambda_{N, n} B_{N}\right) \Lambda_{n}^{N-1} u_{n}\right\|\right\} \leq \tilde{M}
\end{aligned}
$$

for some $\tilde{M}>0$ and $\sup _{n \geq 0}\left\{\sum_{i=1}^{N}\left\|B_{i} \Lambda_{n+1}^{i-1} u_{n+1}\right\|+\tilde{M}\right\} \leq \tilde{M}_{0}$ for some $\tilde{M}_{0}>0$.

Utilizing Proposition 1.1(ii), (v), we deduce that

$$
\begin{aligned}
& \left\|u_{n+1}-u_{n}\right\| \\
& \quad=\left\|\Delta_{n+1}^{M} x_{n+1}-\Delta_{n}^{M} x_{n}\right\|
\end{aligned}
$$




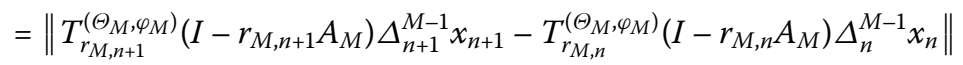

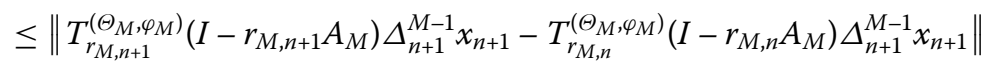

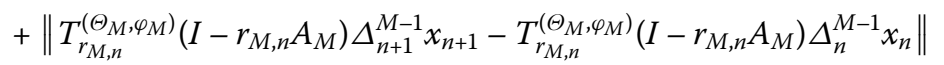

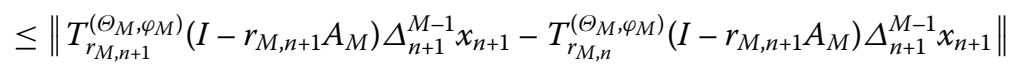

$$
\begin{aligned}
& +\left\|T_{r_{M, n}}^{\left(\Theta_{M}, \varphi_{M}\right)}\left(I-r_{M, n+1} A_{M}\right) \Delta_{n+1}^{M-1} x_{n+1}-T_{r_{M, n}}^{\left(\Theta_{M}, \varphi_{M}\right)}\left(I-r_{M, n} A_{M}\right) \Delta_{n+1}^{M-1} x_{n+1}\right\| \\
& +\left\|\left(I-r_{M, n} A_{M}\right) \Delta_{n+1}^{M-1} x_{n+1}-\left(I-r_{M, n} A_{M}\right) \Delta_{n}^{M-1} x_{n}\right\|
\end{aligned}
$$

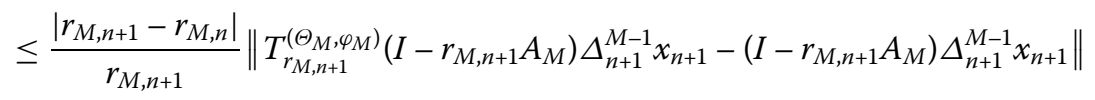

$$
\begin{aligned}
& +\left|r_{M, n+1}-r_{M, n}\right|\left\|A_{M} \Delta_{n+1}^{M-1} x_{n+1}\right\|+\left\|\Delta_{n+1}^{M-1} x_{n+1}-\Delta_{n}^{M-1} x_{n}\right\| \\
& =\left|r_{M, n+1}-r_{M, n}\right|\left[\left\|A_{M} \Delta_{n+1}^{M-1} x_{n+1}\right\|+\frac{1}{r_{M, n+1}} \| T_{r_{M, n+1}^{\left(\Theta_{M}, \varphi_{M}\right)}\left(I-r_{M, n+1} A_{M}\right) \Delta_{n+1}^{M-1} x_{n+1}}\right. \\
& \left.-\left(I-r_{M, n+1} A_{M}\right) \Delta_{n+1}^{M-1} x_{n+1} \|\right]+\left\|\Delta_{n+1}^{M-1} x_{n+1}-\Delta_{n}^{M-1} x_{n}\right\| \\
& \text {. }
\end{aligned}
$$

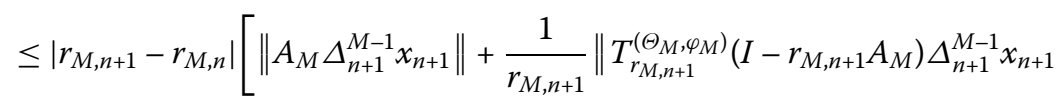

$$
\begin{aligned}
& \left.-\left(I-r_{M, n+1} A_{M}\right) \Delta_{n+1}^{M-1} x_{n+1} \|\right]+\cdots+\left|r_{1, n+1}-r_{1, n}\right|\left[\left\|A_{1} \Delta_{n+1}^{0} x_{n+1}\right\|\right. \\
& \left.+\frac{1}{r_{1, n+1}}\left\|T_{r_{1, n+1}}^{\left(\Theta_{1}, \varphi_{1}\right)}\left(I-r_{1, n+1} A_{1}\right) \Delta_{n+1}^{0} x_{n+1}-\left(I-r_{1, n+1} A_{1}\right) \Delta_{n+1}^{0} x_{n+1}\right\|\right] \\
& +\left\|\Delta_{n+1}^{0} x_{n+1}-\Delta_{n}^{0} x_{n}\right\| \\
& \leq \widetilde{M}_{1} \sum_{k=1}^{M}\left|r_{k, n+1}-r_{k, n}\right|+\left\|x_{n+1}-x_{n}\right\|
\end{aligned}
$$

where $\tilde{M}_{1}>0$ is a constant such that for each $n \geq 0$

$$
\begin{aligned}
& \sum_{k=1}^{M}\left[\left\|A_{k} \Delta_{n+1}^{k-1} x_{n+1}\right\|+\frac{1}{r_{k, n+1}}\left\|T_{r_{k, n+1}}^{\left(\Theta_{k}, \varphi_{k}\right)}\left(I-r_{k, n+1} A_{k}\right) \Delta_{n+1}^{k-1} x_{n+1}-\left(I-r_{k, n+1} A_{k}\right) \Delta_{n+1}^{k-1} x_{n+1}\right\|\right] \\
& \quad \leq \widetilde{M}_{1} .
\end{aligned}
$$

Furthermore, we define $y_{n}=\beta_{n} x_{n}+\left(1-\beta_{n}\right) w_{n}$ for all $n \geq 0$. It follows that

$$
\begin{aligned}
w_{n+1} & -w_{n} \\
= & \frac{y_{n+1}-\beta_{n+1} x_{n+1}}{1-\beta_{n+1}}-\frac{y_{n}-\beta_{n} x_{n}}{1-\beta_{n}} \\
= & \frac{\gamma_{n+1} G v_{n+1}+\delta_{n+1} T G v_{n+1}}{1-\beta_{n+1}}-\frac{\gamma_{n} G v_{n}+\delta_{n} T G v_{n}}{1-\beta_{n}} \\
= & \frac{\gamma_{n+1}\left(G v_{n+1}-G v_{n}\right)+\delta_{n+1}\left(T G v_{n+1}-T G v_{n}\right)}{1-\beta_{n+1}} \\
& +\left(\frac{\gamma_{n+1}}{1-\beta_{n+1}}-\frac{\gamma_{n}}{1-\beta_{n}}\right) G v_{n}+\left(\frac{\delta_{n+1}}{1-\beta_{n+1}}-\frac{\delta_{n}}{1-\beta_{n}}\right) T G v_{n} .
\end{aligned}
$$


Since $\left(\gamma_{n}+\delta_{n}\right) \xi \leq \gamma_{n}$ for all $n \geq 0$, utilizing Lemma 2.2, we have

$$
\left\|\gamma_{n+1}\left(G v_{n+1}-G v_{n}\right)+\delta_{n+1}\left(T G v_{n+1}-T G v_{n}\right)\right\| \leq\left(\gamma_{n+1}+\delta_{n+1}\right)\left\|G v_{n+1}-G v_{n}\right\|
$$

Hence it follows from (3.7)-(3.10) that

$$
\begin{aligned}
\left\|w_{n+1}-w_{n}\right\| & \\
\leq & \frac{\left\|\gamma_{n+1}\left(G v_{n+1}-G v_{n}\right)+\delta_{n+1}\left(T G v_{n+1}-T G v_{n}\right)\right\|}{1-\beta_{n+1}} \\
& +\left|\frac{\gamma_{n+1}}{1-\beta_{n+1}}-\frac{\gamma_{n}}{1-\beta_{n}}\right|\left\|G v_{n}\right\|+\left|\frac{\delta_{n+1}}{1-\beta_{n+1}}-\frac{\delta_{n}}{1-\beta_{n}}\right|\left\|T G v_{n}\right\| \\
\leq & \frac{\left(\gamma_{n+1}+\delta_{n+1}\right)}{1-\beta_{n+1}}\left\|G v_{n+1}-G v_{n}\right\|+\left|\frac{\gamma_{n+1}}{1-\beta_{n+1}}-\frac{\gamma_{n}}{1-\beta_{n}}\right|\left(\left\|G v_{n}\right\|+\left\|T G v_{n}\right\|\right) \\
= & \left\|G v_{n+1}-G v_{n}\right\|+\left|\frac{\gamma_{n+1}}{1-\beta_{n+1}}-\frac{\gamma_{n}}{1-\beta_{n}}\right|\left(\left\|G v_{n}\right\|+\left\|T G v_{n}\right\|\right) \\
\leq & \left\|v_{n+1}-v_{n}\right\|+\left|\frac{\gamma_{n+1}}{1-\beta_{n+1}}-\frac{\gamma_{n}}{1-\beta_{n}}\right|\left(\left\|G v_{n}\right\|+\left\|T G v_{n}\right\|\right) \\
\leq & \tilde{M}_{0} \sum_{i=1}^{N}\left|\lambda_{i, n+1}-\lambda_{i, n}\right|+\left\|u_{n+1}-u_{n}\right\|+\left|\frac{\gamma_{n+1}}{1-\beta_{n+1}}-\frac{\gamma_{n}}{1-\beta_{n}}\right|\left(\left\|G v_{n}\right\|+\left\|T G v_{n}\right\|\right) \\
\leq & \tilde{M}_{0} \sum_{i=1}^{N}\left|\lambda_{i, n+1}-\lambda_{i, n}\right|+\tilde{M} \sum_{k=1}^{M}\left|r_{k, n+1}-r_{k, n}\right|+\left\|x_{n+1}-x_{n}\right\| \\
& +\left|\frac{\gamma_{n+1}}{1-\beta_{n+1}}-\frac{\gamma_{n}}{1-\beta_{n}}\right|\left(\left\|G v_{n}\right\|+\left\|T G v_{n}\right\|\right) .
\end{aligned}
$$

In the meantime, simple calculation shows that

$$
y_{n+1}-y_{n}=\beta_{n}\left(x_{n+1}-x_{n}\right)+\left(1-\beta_{n}\right)\left(w_{n+1}-w_{n}\right)+\left(\beta_{n+1}-\beta_{n}\right)\left(x_{n+1}-w_{n+1}\right) .
$$

So, it follows from (3.11) that

$$
\begin{aligned}
& \left\|y_{n+1}-y_{n}\right\| \\
& \leq \beta_{n}\left\|x_{n+1}-x_{n}\right\|+\left(1-\beta_{n}\right)\left\|w_{n+1}-w_{n}\right\|+\left|\beta_{n+1}-\beta_{n}\right|\left\|x_{n+1}-w_{n+1}\right\| \\
& \leq \beta_{n}\left\|x_{n+1}-x_{n}\right\|+\left(1-\beta_{n}\right)\left[\tilde{M}_{0} \sum_{i=1}^{N}\left|\lambda_{i, n+1}-\lambda_{i, n}\right|+\tilde{M}_{1} \sum_{k=1}^{M}\left|r_{k, n+1}-r_{k, n}\right|+\left\|x_{n+1}-x_{n}\right\|\right. \\
& \left.\quad+\left|\frac{\gamma_{n+1}}{1-\beta_{n+1}}-\frac{\gamma_{n}}{1-\beta_{n}}\right|\left(\left\|G v_{n}\right\|+\left\|T G v_{n}\right\|\right)\right]+\left|\beta_{n+1}-\beta_{n}\right|\left\|x_{n+1}-w_{n+1}\right\| \\
& \leq \quad\left\|x_{n+1}-x_{n}\right\|+\tilde{M}_{0} \sum_{i=1}^{N}\left|\lambda_{i, n+1}-\lambda_{i, n}\right|+\tilde{M}_{1} \sum_{k=1}^{M}\left|r_{k, n+1}-r_{k, n}\right| \\
& \quad+\frac{\left|\gamma_{n+1}-\gamma_{n}\right|\left(1-\beta_{n}\right)+\gamma_{n}\left|\beta_{n+1}-\beta_{n}\right|}{1-\beta_{n+1}}\left(\left\|G v_{n}\right\|+\left\|T G v_{n}\right\|\right) \\
& \quad+\left|\beta_{n+1}-\beta_{n}\right|\left\|x_{n+1}-w_{n+1}\right\|
\end{aligned}
$$




$$
\begin{aligned}
\leq & \left\|x_{n+1}-x_{n}\right\|+\widetilde{M}_{0} \sum_{i=1}^{N}\left|\lambda_{i, n+1}-\lambda_{i, n}\right|+\widetilde{M}_{1} \sum_{k=1}^{M}\left|r_{k, n+1}-r_{k, n}\right| \\
& +\left|\gamma_{n+1}-\gamma_{n}\right| \frac{\left\|G v_{n}\right\|+\left\|T G v_{n}\right\|}{1-b}+\left|\beta_{n+1}-\beta_{n}\right|\left(\left\|x_{n+1}-w_{n+1}\right\|+\frac{\left\|G v_{n}\right\|+\left\|T G v_{n}\right\|}{1-b}\right) \\
\leq & \left\|x_{n+1}-x_{n}\right\|+\widetilde{M}_{2}\left(\sum_{i=1}^{N}\left|\lambda_{i, n+1}-\lambda_{i, n}\right|\right. \\
& \left.+\sum_{k=1}^{M}\left|r_{k, n+1}-r_{k, n}\right|+\left|\gamma_{n+1}-\gamma_{n}\right|+\left|\beta_{n+1}-\beta_{n}\right|\right),
\end{aligned}
$$

where $\sup _{n \geq 0}\left\{\left\|x_{n+1}-w_{n+1}\right\|+\frac{\left\|G v_{n}\right\|+\left\|T G v_{n}\right\|}{1-b}+\widetilde{M}_{0}+\widetilde{M}_{1}\right\} \leq \widetilde{M}_{2}$ for some $\widetilde{M}_{2}>0$.

On the other hand, we define $z_{n}:=\alpha_{n} V x_{n}+\left(1-\alpha_{n}\right) S x_{n}$ for all $n \geq 0$. Then it is well known that $x_{n+1}=\lambda_{n} \gamma z_{n}+\left(I-\lambda_{n} \mu F\right) y_{n}$ for all $n \geq 0$. Simple calculations show that

$$
\left\{\begin{aligned}
z_{n+1}-z_{n}= & \left(\alpha_{n+1}-\alpha_{n}\right)\left(V x_{n}-S x_{n}\right)+\alpha_{n+1}\left(V x_{n+1}-V x_{n}\right) \\
+ & \left(1-\alpha_{n+1}\right)\left(S x_{n+1}-S x_{n}\right), \\
x_{n+2}-x_{n+1}= & \left(\lambda_{n+1}-\lambda_{n}\right)\left(\gamma z_{n}-\mu F y_{n}\right)+\lambda_{n+1} \gamma\left(z_{n+1}-z_{n}\right) \\
& +\left(I-\lambda_{n+1} \mu F\right) y_{n+1}-\left(I-\lambda_{n+1} \mu F\right) y_{n} .
\end{aligned}\right.
$$

Since $V$ is a $\rho$-contraction with coefficient $\rho \in[0,1)$ and $S$ is a nonexpansive mapping, we conclude that

$$
\begin{aligned}
\left\|z_{n+1}-z_{n}\right\| \leq & \left|\alpha_{n+1}-\alpha_{n}\right|\left\|V x_{n}-S x_{n}\right\|+\alpha_{n+1}\left\|V x_{n+1}-V x_{n}\right\| \\
& +\left(1-\alpha_{n+1}\right)\left\|S x_{n+1}-S x_{n}\right\| \\
\leq & \left|\alpha_{n+1}-\alpha_{n}\right|\left\|V x_{n}-S x_{n}\right\|+\alpha_{n+1} \rho\left\|x_{n+1}-x_{n}\right\|+\left(1-\alpha_{n+1}\right)\left\|x_{n+1}-x_{n}\right\| \\
= & \left(1-\alpha_{n+1}(1-\rho)\right)\left\|x_{n+1}-x_{n}\right\|+\left|\alpha_{n+1}-\alpha_{n}\right|\left\|V x_{n}-S x_{n}\right\|,
\end{aligned}
$$

which together with (3.12) and $0 \leq \gamma<\tau$ implies that

$$
\begin{aligned}
& \left\|x_{n+2}-x_{n+1}\right\| \\
& \leq\left|\lambda_{n+1}-\lambda_{n}\right|\left\|\gamma z_{n}-\mu F y_{n}\right\|+\lambda_{n+1} \gamma\left\|z_{n+1}-z_{n}\right\| \\
& +\left\|\left(I-\lambda_{n+1} \mu F\right) y_{n+1}-\left(I-\lambda_{n+1} \mu F\right) y_{n}\right\| \\
& \leq\left|\lambda_{n+1}-\lambda_{n}\right|\left\|\gamma z_{n}-\mu F y_{n}\right\|+\lambda_{n+1} \gamma\left\|z_{n+1}-z_{n}\right\|+\left(1-\lambda_{n+1} \tau\right)\left\|y_{n+1}-y_{n}\right\| \\
& \leq\left|\lambda_{n+1}-\lambda_{n}\right|\left\|\gamma z_{n}-\mu F y_{n}\right\|+\lambda_{n+1} \gamma\left[\left(1-\alpha_{n+1}(1-\rho)\right)\left\|x_{n+1}-x_{n}\right\|\right. \\
& \left.+\left|\alpha_{n+1}-\alpha_{n}\right|\left\|V x_{n}-S x_{n}\right\|\right]+\left(1-\lambda_{n+1} \tau\right)\left[\left\|x_{n+1}-x_{n}\right\|+\tilde{M}_{2}\left(\sum_{i=1}^{N}\left|\lambda_{i, n+1}-\lambda_{i, n}\right|\right.\right. \\
& \left.\left.+\sum_{k=1}^{M}\left|r_{k, n+1}-r_{k, n}\right|+\left|\gamma_{n+1}-\gamma_{n}\right|+\left|\beta_{n+1}-\beta_{n}\right|\right)\right] \\
& \leq\left(1-\lambda_{n+1}(\tau-\gamma)\right)\left\|x_{n+1}-x_{n}\right\|+\left|\lambda_{n+1}-\lambda_{n}\right|\left\|\gamma z_{n}-\mu F y_{n}\right\|+\left|\alpha_{n+1}-\alpha_{n}\right|\left\|V x_{n}-S x_{n}\right\| \\
& +\widetilde{M}_{2}\left(\sum_{i=1}^{N}\left|\lambda_{i, n+1}-\lambda_{i, n}\right|+\sum_{k=1}^{M}\left|r_{k, n+1}-r_{k, n}\right|+\left|\gamma_{n+1}-\gamma_{n}\right|+\left|\beta_{n+1}-\beta_{n}\right|\right)
\end{aligned}
$$




$$
\begin{aligned}
\leq & \left(1-\lambda_{n+1}(\tau-\gamma)\right)\left\|x_{n+1}-x_{n}\right\|+\tilde{M}_{3}\left\{\sum_{i=1}^{N}\left|\lambda_{i, n+1}-\lambda_{i, n}\right|+\sum_{k=1}^{M}\left|r_{k, n+1}-r_{k, n}\right|\right. \\
& \left.+\left|\lambda_{n+1}-\lambda_{n}\right|+\left|\alpha_{n+1}-\alpha_{n}\right|+\left|\beta_{n+1}-\beta_{n}\right|+\left|\gamma_{n+1}-\gamma_{n}\right|\right\},
\end{aligned}
$$

where $\sup _{n \geq 0}\left\{\left\|\gamma z_{n}-\mu F y_{n}\right\|+\left\|V x_{n}-S x_{n}\right\|+\widetilde{M}_{2}\right\} \leq \widetilde{M}_{3}$ for some $\widetilde{M}_{3}>0$. Consequently,

$$
\begin{aligned}
\frac{\left\|x_{n+1}-x_{n}\right\|}{\alpha_{n}} & \left(1-\lambda_{n}(\tau-\gamma)\right) \frac{|| x_{n}-x_{n-1} \|}{\alpha_{n}}+\tilde{M}_{3}\left\{\sum_{i=1}^{N} \frac{\left|\lambda_{i, n}-\lambda_{i, n-1}\right|}{\alpha_{n}}+\sum_{k=1}^{M} \frac{\left|r_{k, n}-r_{k, n-1}\right|}{\alpha_{n}}\right. \\
& \left.+\frac{\left|\lambda_{n}-\lambda_{n-1}\right|}{\alpha_{n}}+\frac{\left|\alpha_{n}-\alpha_{n-1}\right|}{\alpha_{n}}+\frac{\left|\beta_{n}-\beta_{n-1}\right|}{\alpha_{n}}+\frac{\left|\gamma_{n}-\gamma_{n-1}\right|}{\alpha_{n}}\right\} \\
= & \left(1-\lambda_{n}(\tau-\gamma)\right) \frac{|| x_{n}-x_{n-1} \mid}{\alpha_{n-1}}+\left(1-\lambda_{n}(\tau-\gamma)\right) \| x_{n}-x_{n-1}||\left(\frac{1}{\alpha_{n}}-\frac{1}{\alpha_{n-1}}\right) \\
& +\tilde{M}_{3}\left\{\sum_{i=1}^{N} \frac{\left|\lambda_{i, n}-\lambda_{i, n-1}\right|}{\alpha_{n}}+\sum_{k=1}^{M} \frac{\left|r_{k, n}-r_{k, n-1}\right|}{\alpha_{n}}+\frac{\left|\lambda_{n}-\lambda_{n-1}\right|}{\alpha_{n}}\right. \\
& \left.+\frac{\left|\alpha_{n}-\alpha_{n-1}\right|}{\alpha_{n}}+\frac{\left|\beta_{n}-\beta_{n-1}\right|}{\alpha_{n}}+\frac{\left|\gamma_{n}-\gamma_{n-1}\right|}{\alpha_{n}}\right\} \\
\leq & \left(1-\lambda_{n}(\tau-\gamma)\right) \frac{|| x_{n}-x_{n-1} \|}{\alpha_{n-1}}+\lambda_{n}(\tau-\gamma) \cdot \frac{\tilde{M}_{4}}{\tau-\gamma}\left\{\frac{1}{\lambda_{n}}\left|\frac{1}{\alpha_{n}}-\frac{1}{\alpha_{n-1}}\right|\right. \\
& +\sum_{i=1}^{N} \frac{\left|\lambda_{i, n}-\lambda_{i, n-1}\right|}{\lambda_{n} \alpha_{n}}+\sum_{k=1}^{M} \frac{\left|r_{k, n}-r_{k, n-1}\right|}{\lambda_{n} \alpha_{n}}+\frac{1}{\alpha_{n}}\left|1-\frac{\lambda_{n-1}}{\lambda_{n}}\right| \\
& \left.+\frac{1}{\lambda_{n}}\left|1-\frac{\alpha_{n-1}}{\alpha_{n}}\right|+\frac{\left|\beta_{n}-\beta_{n-1}\right|}{\lambda_{n} \alpha_{n}}+\frac{\left|\gamma_{n}-\gamma_{n-1}\right|}{\lambda_{n} \alpha_{n}}\right\}
\end{aligned}
$$

where $\sup _{n \geq 1}\left\{\left\|x_{n}-x_{n-1}\right\|+\widetilde{M}_{3}\right\} \leq \widetilde{M}_{4}$ for some $\widetilde{M}_{4}>0$. From conditions (i)-(iv) it follows that $\sum_{n=0}^{\infty} \lambda_{n}(\tau-\gamma)=\infty$ and

$$
\begin{aligned}
& \lim _{n \rightarrow \infty} \frac{\tilde{M}_{4}}{\tau-\gamma}\left\{\frac{1}{\lambda_{n}}\left|\frac{1}{\alpha_{n}}-\frac{1}{\alpha_{n-1}}\right|+\sum_{i=1}^{N} \frac{\left|\lambda_{i, n}-\lambda_{i, n-1}\right|}{\lambda_{n} \alpha_{n}}+\sum_{k=1}^{M} \frac{\left|r_{k, n}-r_{k, n-1}\right|}{\lambda_{n} \alpha_{n}}\right. \\
& \left.+\frac{1}{\alpha_{n}}\left|1-\frac{\lambda_{n-1}}{\lambda_{n}}\right|+\frac{1}{\lambda_{n}}\left|1-\frac{\alpha_{n-1}}{\alpha_{n}}\right|+\frac{\left|\beta_{n}-\beta_{n-1}\right|}{\lambda_{n} \alpha_{n}}+\frac{\left|\gamma_{n}-\gamma_{n-1}\right|}{\lambda_{n} \alpha_{n}}\right\}=0 .
\end{aligned}
$$

Thus, utilizing Lemma 2.12, we immediately conclude that

$$
\lim _{n \rightarrow \infty} \frac{\left\|x_{n+1}-x_{n}\right\|}{\alpha_{n}}=0
$$

So, from $\alpha_{n} \rightarrow 0$ it follows that

$$
\lim _{n \rightarrow \infty}\left\|x_{n+1}-x_{n}\right\|=0
$$


Step 3. We prove that $\lim _{n \rightarrow \infty}\left\|x_{n}-u_{n}\right\|=0, \lim _{n \rightarrow \infty}\left\|x_{n}-v_{n}\right\|=0, \lim _{n \rightarrow \infty}\left\|v_{n}-G v_{n}\right\|=0$ and $\lim _{n \rightarrow \infty}\left\|v_{n}-T v_{n}\right\|=0$.

Indeed, utilizing Lemmas 2.8 and 2.9(b), from (3.1), (3.4)-(3.5), and $0 \leq \gamma<\tau$ we deduce that

$$
\begin{aligned}
\left\|y_{n}-p\right\|^{2} & \\
= & \left\|\beta_{n} x_{n}+\gamma_{n} G v_{n}+\delta_{n} T G v_{n}-p\right\|^{2} \\
= & \left\|\beta_{n}\left(x_{n}-p\right)+\left(1-\beta_{n}\right)\left(\frac{\gamma_{n} G v_{n}+\delta_{n} T G v_{n}}{1-\beta_{n}}-p\right)\right\|^{2} \\
= & \beta_{n}\left\|x_{n}-p\right\|^{2}+\left(1-\beta_{n}\right)\left\|\frac{\gamma_{n} G v_{n}+\delta_{n} T G v_{n}}{1-\beta_{n}}-p\right\|^{2} \\
& -\beta_{n}\left(1-\beta_{n}\right)\left\|\frac{\gamma_{n} G v_{n}+\delta_{n} T G v_{n}}{1-\beta_{n}}-x_{n}\right\|^{2} \\
= & \beta_{n}\left\|x_{n}-p\right\|^{2}+\left(1-\beta_{n}\right)\left\|\frac{\gamma_{n}\left(G v_{n}-p\right)+\delta_{n}\left(T G v_{n}-p\right)}{1-\beta_{n}}\right\|^{2}-\beta_{n}\left(1-\beta_{n}\right)\left\|\frac{y_{n}-x_{n}}{1-\beta_{n}}\right\|^{2} \\
\leq & \beta_{n}\left\|x_{n}-p\right\|^{2}+\left(1-\beta_{n}\right) \frac{\left(\gamma_{n}+\delta_{n}\right)^{2}\left\|G v_{n}-p\right\|^{2}}{\left(1-\beta_{n}\right)^{2}}-\frac{\beta_{n}}{1-\beta_{n}}\left\|y_{n}-x_{n}\right\|^{2} \\
= & \beta_{n}\left\|x_{n}-p\right\|^{2}+\left(1-\beta_{n}\right)\left\|G v_{n}-p\right\|^{2}-\frac{\beta_{n}}{1-\beta_{n}}\left\|y_{n}-x_{n}\right\|^{2} \\
\leq & \beta_{n}\left\|x_{n}-p\right\|^{2}+\left(1-\beta_{n}\right)\left\|v_{n}-p\right\|^{2}-\frac{\beta_{n}}{1-\beta_{n}}\left\|y_{n}-x_{n}\right\|^{2} \\
\leq & \beta_{n}\left\|x_{n}-p\right\|^{2}+\left(1-\beta_{n}\right)\left\|x_{n}-p\right\|^{2}-\frac{\beta_{n}}{1-\beta_{n}}\left\|y_{n}-x_{n}\right\|^{2} \\
= & \left\|x_{n}-p\right\|^{2}-\frac{\beta_{n}}{1-\beta_{n}}\left\|y_{n}-x_{n}\right\|^{2},
\end{aligned}
$$

and hence

$$
\begin{aligned}
\left\|x_{n+1}-p\right\|^{2} & =\left\|\lambda_{n} \gamma\left(\alpha_{n} V x_{n}+\left(1-\alpha_{n}\right) S x_{n}\right)+\left(I-\lambda_{n} \mu F\right) y_{n}-p\right\|^{2} \\
= & \left\|\lambda_{n} \gamma\left(\alpha_{n} V x_{n}+\left(1-\alpha_{n}\right) S x_{n}\right)-\lambda_{n} \mu F p+\left(I-\lambda_{n} \mu F\right) y_{n}-\left(I-\lambda_{n} \mu F\right) p\right\|^{2} \\
= & \left\|\lambda_{n}\left[\alpha_{n}\left(\gamma V x_{n}-\mu F p\right)+\left(1-\alpha_{n}\right)\left(\gamma S x_{n}-\mu F p\right)\right]+\left(I-\lambda_{n} \mu F\right) y_{n}-\left(I-\lambda_{n} \mu F\right) p\right\|^{2} \\
= & \| \lambda_{n}\left[\alpha_{n}\left(\gamma V x_{n}-\gamma V p\right)+\left(1-\alpha_{n}\right)\left(\gamma S x_{n}-\gamma S p\right)\right]+\left(I-\lambda_{n} \mu F\right) y_{n}-\left(I-\lambda_{n} \mu F\right) p \\
& +\lambda_{n}\left[\alpha_{n}(\gamma V p-\mu F p)+\left(1-\alpha_{n}\right)(\gamma S p-\mu F p)\right] \|^{2} \\
\leq & \left\|\lambda_{n}\left[\alpha_{n}\left(\gamma V x_{n}-\gamma V p\right)+\left(1-\alpha_{n}\right)\left(\gamma S x_{n}-\gamma S p\right)\right]+\left(I-\lambda_{n} \mu F\right) y_{n}-\left(I-\lambda_{n} \mu F\right) p\right\|^{2} \\
& +2 \lambda_{n} \alpha_{n}\left((\gamma V p-\mu F p), x_{n+1}-p\right\rangle+2 \lambda_{n}\left(1-\alpha_{n}\right)\left\langle(\gamma S p-\mu F p), x_{n+1}-p\right\rangle \\
\leq & {\left[\lambda_{n}\left\|\alpha_{n}\left(\gamma V x_{n}-\gamma V p\right)+\left(1-\alpha_{n}\right)\left(\gamma S x_{n}-\gamma S p\right)\right\|+\left\|\left(I-\lambda_{n} \mu F\right) y_{n}-\left(I-\lambda_{n} \mu F\right) p\right\|\right]^{2} } \\
& +2 \lambda_{n} \alpha_{n}\left\langle(\gamma V p-\mu F p), x_{n+1}-p\right\rangle+2 \lambda_{n}\left(1-\alpha_{n}\right)\left\langle(\gamma S p-\mu F p), x_{n+1}-p\right\rangle \\
\leq & {\left[\lambda_{n}\left(\alpha_{n} \gamma \rho\left\|x_{n}-p\right\|+\left(1-\alpha_{n}\right) \gamma\left\|x_{n}-p\right\|\right)+\left(1-\lambda_{n} \tau\right)\left\|y_{n}-p\right\|\right]^{2} } \\
& +2 \lambda_{n} \alpha_{n}\left((\gamma V p-\mu F p), x_{n+1}-p\right\rangle+2\left(1-\alpha_{n}\right) \lambda_{n}\left\langle(\gamma S p-\mu F p), x_{n+1}-p\right\rangle
\end{aligned}
$$




$$
\begin{aligned}
= & {\left[\lambda_{n}\left(1-\alpha_{n}(1-\rho)\right) \gamma\left\|x_{n}-p\right\|+\left(1-\lambda_{n} \tau\right)\left\|y_{n}-p\right\|\right]^{2} } \\
& +2 \lambda_{n} \alpha_{n}\left\langle(\gamma V p-\mu F p), x_{n+1}-p\right\rangle+2 \lambda_{n}\left(1-\alpha_{n}\right)\left\langle(\gamma S p-\mu F p), x_{n+1}-p\right\rangle \\
\leq & {\left[\lambda_{n} \gamma\left\|x_{n}-p\right\|+\left(1-\lambda_{n} \tau\right)\left\|y_{n}-p\right\|\right]^{2} } \\
& +2 \lambda_{n} \alpha_{n}\left\langle(\gamma V p-\mu F p), x_{n+1}-p\right\rangle+2 \lambda_{n}\left(1-\alpha_{n}\right)\left\langle(\gamma S p-\mu F p), x_{n+1}-p\right\rangle \\
= & {\left[\lambda_{n} \tau \cdot \frac{\gamma}{\tau}\left\|x_{n}-p\right\|+\left(1-\lambda_{n} \tau\right)\left\|y_{n}-p\right\|\right]^{2} } \\
& +2 \lambda_{n} \alpha_{n}\left\langle(\gamma V p-\mu F p), x_{n+1}-p\right\rangle+2 \lambda_{n}\left(1-\alpha_{n}\right)\left\langle(\gamma S p-\mu F p), x_{n+1}-p\right\rangle \\
\leq & \lambda_{n} \frac{\gamma^{2}}{\tau}\left\|x_{n}-p\right\|+\left(1-\lambda_{n} \tau\right)\left\|y_{n}-p\right\|^{2} \\
& +2 \lambda_{n} \alpha_{n}\left\langle(\gamma V p-\mu F p), x_{n+1}-p\right\rangle+2 \lambda_{n}\left(1-\alpha_{n}\right)\left\langle(\gamma S p-\mu F p), x_{n+1}-p\right\rangle \\
\leq & \lambda_{n} \frac{\gamma^{2}}{\tau}\left\|x_{n}-p\right\|+\left(1-\lambda_{n} \tau\right)\left[\left\|x_{n}-p\right\|^{2}-\frac{\beta_{n}}{1-\beta_{n}}\left\|y_{n}-x_{n}\right\|^{2}\right] \\
& +2 \lambda_{n} \alpha_{n}\left\langle(\gamma V p-\mu F p), x_{n+1}-p\right\rangle+2 \lambda_{n}\left(1-\alpha_{n}\right)\left\langle(\gamma S p-\mu F p), x_{n+1}-p\right\rangle \\
= & \left(1-\lambda_{n} \frac{\tau^{2}-\gamma^{2}}{\tau}\right)\left\|x_{n}-p\right\|^{2}-\frac{\beta_{n}\left(1-\lambda_{n} \tau\right)}{1-\beta_{n}}\left\|y_{n}-x_{n}\right\|^{2} \\
& +2 \lambda_{n} \alpha_{n}\left\langle(\gamma V p-\mu F p), x_{n+1}-p\right\rangle+2 \lambda_{n}\left(1-\alpha_{n}\right)\left\langle(\gamma S p-\mu F p), x_{n+1}-p\right\rangle \\
\leq & \left\|x_{n}-p\right\|^{2}-\frac{\beta_{n}\left(1-\lambda \lambda_{n} \tau\right)}{1-\beta_{n}}\left\|y_{n}-x_{n}\right\|^{2} \\
& +2 \lambda_{n} \alpha_{n}\|\gamma V p-\mu F p\|\left\|x_{n+1}-p\right\|+2 \lambda_{n}\|\gamma S p-\mu F p\|\left\|x_{n+1}-p\right\|,
\end{aligned}
$$

which together with $\left\{\beta_{n}\right\} \subset[a, b] \subset(0,1)$, immediately yields

$$
\begin{aligned}
\frac{a\left(1-\lambda_{n} \tau\right)}{1-a}\left\|y_{n}-x_{n}\right\|^{2} \leq & \frac{\beta_{n}\left(1-\lambda_{n} \tau\right)}{1-\beta_{n}}\left\|y_{n}-x_{n}\right\|^{2} \\
\leq & \left\|x_{n}-p\right\|^{2}-\left\|x_{n+1}-p\right\|^{2}+2 \lambda_{n} \alpha_{n}\|\gamma V p-\mu F p\|\left\|x_{n+1}-p\right\| \\
& +2 \lambda_{n}\|\gamma S p-\mu F p\|\left\|x_{n+1}-p\right\| \\
\leq & \left\|x_{n}-x_{n+1}\right\|\left(\left\|x_{n}-p\right\|+\left\|x_{n+1}-p\right\|\right) \\
& +2 \lambda_{n} \alpha_{n}\|\gamma V p-\mu F p\|\left\|x_{n+1}-p\right\| \\
& +2 \lambda_{n}\|\gamma S p-\mu F p\|\left\|x_{n+1}-p\right\| .
\end{aligned}
$$

Since $\lambda_{n} \rightarrow 0, \alpha_{n} \rightarrow 0,\left\|x_{n+1}-x_{n}\right\| \rightarrow 0$, and $\left\{x_{n}\right\}$ is bounded, we have

$$
\lim _{n \rightarrow \infty}\left\|y_{n}-x_{n}\right\|=0 \text {. }
$$

Observe that

$$
\begin{aligned}
\left\|\Delta_{n}^{k} x_{n}-p\right\|^{2} & =\left\|T_{r_{k, n}}^{\left(\Theta_{k}, \varphi_{k}\right)}\left(I-r_{k, n} A_{k}\right) \Delta_{n}^{k-1} x_{n}-T_{r_{k, n}}^{\left(\Theta_{k}, \varphi_{k}\right)}\left(I-r_{k, n} A_{k}\right) p\right\|^{2} \\
& \leq\left\|\left(I-r_{k, n} A_{k}\right) \Delta_{n}^{k-1} x_{n}-\left(I-r_{k, n} A_{k}\right) p\right\|^{2} \\
& \leq\left\|\Delta_{n}^{k-1} x_{n}-p\right\|^{2}+r_{k, n}\left(r_{k, n}-2 \mu_{k}\right)\left\|A_{k} \Delta_{n}^{k-1} x_{n}-A_{k} p\right\|^{2} \\
& \leq\left\|x_{n}-p\right\|^{2}+r_{k, n}\left(r_{k, n}-2 \mu_{k}\right)\left\|A_{k} \Delta_{n}^{k-1} x_{n}-A_{k} p\right\|^{2}
\end{aligned}
$$


and

$$
\begin{aligned}
\left\|\Lambda_{n}^{i} u_{n}-p\right\|^{2} & =\left\|J_{R_{i}, \lambda_{i, n}}\left(I-\lambda_{i, n} B_{i}\right) \Lambda_{n}^{i-1} u_{n}-J_{R_{i}, \lambda_{i, n}}\left(I-\lambda_{i, n} B_{i}\right) p\right\|^{2} \\
& \leq\left\|\left(I-\lambda_{i, n} B_{i}\right) \Lambda_{n}^{i-1} u_{n}-\left(I-\lambda_{i, n} B_{i}\right) p\right\|^{2} \\
& \leq\left\|\Lambda_{n}^{i-1} u_{n}-p\right\|^{2}+\lambda_{i, n}\left(\lambda_{i, n}-2 \eta_{i}\right)\left\|B_{i} \Lambda_{n}^{i-1} u_{n}-B_{i} p\right\|^{2} \\
& \leq\left\|u_{n}-p\right\|^{2}+\lambda_{i, n}\left(\lambda_{i, n}-2 \eta_{i}\right)\left\|B_{i} \Lambda_{n}^{i-1} u_{n}-B_{i} p\right\|^{2} \\
& \leq\left\|x_{n}-p\right\|^{2}+\lambda_{i, n}\left(\lambda_{i, n}-2 \eta_{i}\right)\left\|B_{i} \Lambda_{n}^{i-1} u_{n}-B_{i} p\right\|^{2}
\end{aligned}
$$

for $i \in\{1,2, \ldots, N\}$ and $k \in\{1,2, \ldots, M\}$. Combining (3.15), (3.18), and (3.19), we get

$$
\begin{aligned}
\left\|y_{n}-p\right\|^{2} \leq & \beta_{n}\left\|x_{n}-p\right\|^{2}+\left(1-\beta_{n}\right)\left\|v_{n}-p\right\|^{2}-\frac{\beta_{n}}{1-\beta_{n}}\left\|y_{n}-x_{n}\right\|^{2} \\
\leq & \beta_{n}\left\|x_{n}-p\right\|^{2}+\left(1-\beta_{n}\right)\left\|v_{n}-p\right\|^{2} \\
\leq & \beta_{n}\left\|x_{n}-p\right\|^{2}+\left(1-\beta_{n}\right)\left\|\Lambda_{n}^{i} u_{n}-p\right\|^{2} \\
\leq & \beta_{n}\left\|x_{n}-p\right\|^{2}+\left(1-\beta_{n}\right)\left[\left\|u_{n}-p\right\|^{2}+\lambda_{i, n}\left(\lambda_{i, n}-2 \eta_{i}\right)\left\|B_{i} \Lambda_{n}^{i-1} u_{n}-B_{i} p\right\|^{2}\right] \\
\leq & \beta_{n}\left\|x_{n}-p\right\|^{2}+\left(1-\beta_{n}\right)\left[\left\|\Delta_{n}^{k} x_{n}-p\right\|^{2}+\lambda_{i, n}\left(\lambda_{i, n}-2 \eta_{i}\right)\left\|B_{i} \Lambda_{n}^{i-1} u_{n}-B_{i} p\right\|^{2}\right] \\
\leq & \beta_{n}\left\|x_{n}-p\right\|^{2}+\left(1-\beta_{n}\right)\left[\left\|x_{n}-p\right\|^{2}+r_{k, n}\left(r_{k, n}-2 \mu_{k}\right)\left\|A_{k} \Delta_{n}^{k-1} x_{n}-A_{k} p\right\|^{2}\right. \\
& \left.+\lambda_{i, n}\left(\lambda_{i, n}-2 \eta_{i}\right)\left\|B_{i} \Lambda_{n}^{i-1} u_{n}-B_{i} p\right\|^{2}\right] \\
= & \left\|x_{n}-p\right\|^{2}+\left(1-\beta_{n}\right)\left[r_{k, n}\left(r_{k, n}-2 \mu_{k}\right)\left\|A_{k} \Delta_{n}^{k-1} x_{n}-A_{k} p\right\|^{2}\right. \\
& \left.+\lambda_{i, n}\left(\lambda_{i, n}-2 \eta_{i}\right)\left\|B_{i} \Lambda_{n}^{i-1} u_{n}-B_{i} p\right\|^{2}\right],
\end{aligned}
$$

which immediately leads to

$$
\begin{aligned}
& \left(1-\beta_{n}\right)\left[r_{k, n}\left(2 \mu_{k}-r_{k, n}\right)\left\|A_{k} \Delta_{n}^{k-1} x_{n}-A_{k} p\right\|^{2}+\lambda_{i, n}\left(2 \eta_{i}-\lambda_{i, n}\right)\left\|B_{i} \Lambda_{n}^{i-1} u_{n}-B_{i} p\right\|^{2}\right] \\
& \quad \leq\left\|x_{n}-p\right\|^{2}-\left\|y_{n}-p\right\|^{2} \\
& \quad \leq\left\|x_{n}-y_{n}\right\|\left(\left\|x_{n}-p\right\|+\left\|y_{n}-p\right\|\right) .
\end{aligned}
$$

Since $\left\|x_{n}-y_{n}\right\| \rightarrow 0,\left\{\beta_{n}\right\} \subset[a, b] \subset(0,1),\left\{\lambda_{i, n}\right\} \subset\left[a_{i}, b_{i}\right] \subset\left(0,2 \eta_{i}\right),\left\{r_{k, n}\right\} \subset\left[c_{k}, d_{k}\right] \subset$ $\left(0,2 \mu_{k}\right), i \in\{1,2, \ldots, N\}, k \in\{1,2, \ldots, M\}$, and $\left\{x_{n}\right\},\left\{y_{n}\right\}$ are bounded sequences, we have

$$
\lim _{n \rightarrow \infty}\left\|A_{k} \Delta_{n}^{k-1} x_{n}-A_{k} p\right\|=0 \quad \text { and } \quad \lim _{n \rightarrow \infty}\left\|B_{i} \Lambda_{n}^{i-1} u_{n}-B_{i} p\right\|=0
$$

for all $k \in\{1,2, \ldots, M\}$ and $i \in\{1,2, \ldots, N\}$.

Furthermore, by Proposition 1.1(ii) and Lemma 2.9(a), we have

$$
\begin{aligned}
\| & \Delta_{n}^{k} x_{n}-p \|^{2} \\
& =\left\|T_{r_{k, n}}^{\left(\Theta_{k}, \varphi_{k}\right)}\left(I-r_{k, n} A_{k}\right) \Delta_{n}^{k-1} x_{n}-T_{r_{k, n}}^{\left(\Theta_{k}, \varphi_{k}\right)}\left(I-r_{k, n} A_{k}\right) p\right\|^{2} \\
& \leq\left\langle\left(I-r_{k, n} A_{k}\right) \Delta_{n}^{k-1} x_{n}-\left(I-r_{k, n} A_{k}\right) p, \Delta_{n}^{k} x_{n}-p\right\rangle \\
& =\frac{1}{2}\left(\left\|\left(I-r_{k, n} A_{k}\right) \Delta_{n}^{k-1} x_{n}-\left(I-r_{k, n} A_{k}\right) p\right\|^{2}+\left\|\Delta_{n}^{k} x_{n}-p\right\|^{2}\right.
\end{aligned}
$$




$$
\begin{aligned}
& \left.-\left\|\left(I-r_{k, n} A_{k}\right) \Delta_{n}^{k-1} x_{n}-\left(I-r_{k, n} A_{k}\right) p-\left(\Delta_{n}^{k} x_{n}-p\right)\right\|^{2}\right) \\
\leq & \frac{1}{2}\left(\left\|\Delta_{n}^{k-1} x_{n}-p\right\|^{2}+\left\|\Delta_{n}^{k} x_{n}-p\right\|^{2}-\left\|\Delta_{n}^{k-1} x_{n}-\Delta_{n}^{k} x_{n}-r_{k, n}\left(A_{k} \Delta_{n}^{k-1} x_{n}-A_{k} p\right)\right\|^{2}\right),
\end{aligned}
$$

which implies that

$$
\begin{aligned}
&\left\|\Delta_{n}^{k} x_{n}-p\right\|^{2} \\
& \leq\left\|\Delta_{n}^{k-1} x_{n}-p\right\|^{2}-\left\|\Delta_{n}^{k-1} x_{n}-\Delta_{n}^{k} x_{n}-r_{k, n}\left(A_{k} \Delta_{n}^{k-1} x_{n}-A_{k} p\right)\right\|^{2} \\
&=\left\|\Delta_{n}^{k-1} x_{n}-p\right\|^{2}-\left\|\Delta_{n}^{k-1} x_{n}-\Delta_{n}^{k} x_{n}\right\|^{2}-r_{k, n}^{2}\left\|A_{k} \Delta_{n}^{k-1} x_{n}-A_{k} p\right\|^{2} \\
& \quad+2 r_{k, n}\left\langle\Delta_{n}^{k-1} x_{n}-\Delta_{n}^{k} x_{n}, A_{k} \Delta_{n}^{k-1} x_{n}-A_{k} p\right\rangle \\
& \leq\left\|\Delta_{n}^{k-1} x_{n}-p\right\|^{2}-\left\|\Delta_{n}^{k-1} x_{n}-\Delta_{n}^{k} x_{n}\right\|^{2}+2 r_{k, n}\left\|\Delta_{n}^{k-1} x_{n}-\Delta_{n}^{k} x_{n}\right\|\left\|A_{k} \Delta_{n}^{k-1} x_{n}-A_{k} p\right\| \\
& \leq\left\|x_{n}-p\right\|^{2}-\left\|\Delta_{n}^{k-1} x_{n}-\Delta_{n}^{k} x_{n}\right\|^{2}+2 r_{k, n}\left\|\Delta_{n}^{k-1} x_{n}-\Delta_{n}^{k} x_{n}\right\|\left\|A_{k} \Delta_{n}^{k-1} x_{n}-A_{k} p\right\| . \quad \text { (3.21) }
\end{aligned}
$$

By Lemma 2.9(a) and Lemma 2.3, we obtain

$$
\begin{aligned}
&\left\|\Lambda_{n}^{i} u_{n}-p\right\|^{2} \\
&=\left\|J_{R_{i}, \lambda_{i, n}}\left(I-\lambda_{i, n} B_{i}\right) \Lambda_{n}^{i-1} u_{n}-J_{R_{i}, \lambda_{i, n}}\left(I-\lambda_{i, n} B_{i}\right) p\right\|^{2} \\
& \leq\left\langle\left(I-\lambda_{i, n} B_{i}\right) \Lambda_{n}^{i-1} u_{n}-\left(I-\lambda_{i, n} B_{i}\right) p, \Lambda_{n}^{i} u_{n}-p\right\rangle \\
&= \frac{1}{2}\left(\left\|\left(I-\lambda_{i, n} B_{i}\right) \Lambda_{n}^{i-1} u_{n}-\left(I-\lambda_{i, n} B_{i}\right) p\right\|^{2}+\left\|\Lambda_{n}^{i} u_{n}-p\right\|^{2}\right. \\
&\left.\quad-\left\|\left(I-\lambda_{i, n} B_{i}\right) \Lambda_{n}^{i-1} u_{n}-\left(I-\lambda_{i, n} B_{i}\right) p-\left(\Lambda_{n}^{i} u_{n}-p\right)\right\|^{2}\right) \\
& \leq \frac{1}{2}\left(\left\|\Lambda_{n}^{i-1} u_{n}-p\right\|^{2}+\left\|\Lambda_{n}^{i} u_{n}-p\right\|^{2}-\left\|\Lambda_{n}^{i-1} u_{n}-\Lambda_{n}^{i} u_{n}-\lambda_{i, n}\left(B_{i} \Lambda_{n}^{i-1} u_{n}-B_{i} p\right)\right\|^{2}\right) \\
& \leq \frac{1}{2}\left(\left\|u_{n}-p\right\|^{2}+\left\|\Lambda_{n}^{i} u_{n}-p\right\|^{2}-\left\|\Lambda_{n}^{i-1} u_{n}-\Lambda_{n}^{i} u_{n}-\lambda_{i, n}\left(B_{i} \Lambda_{n}^{i-1} u_{n}-B_{i} p\right)\right\|^{2}\right) \\
& \leq \frac{1}{2}\left(\left\|x_{n}-p\right\|^{2}+\left\|\Lambda_{n}^{i} u_{n}-p\right\|^{2}-\left\|\Lambda_{n}^{i-1} u_{n}-\Lambda_{n}^{i} u_{n}-\lambda_{i, n}\left(B_{i} \Lambda_{n}^{i-1} u_{n}-B_{i} p\right)\right\|^{2}\right),
\end{aligned}
$$

which immediately leads to

$$
\begin{aligned}
& \left\|\Lambda_{n}^{i} u_{n}-p\right\|^{2} \\
& \quad \leq\left\|x_{n}-p\right\|^{2}-\left\|\Lambda_{n}^{i-1} u_{n}-\Lambda_{n}^{i} u_{n}-\lambda_{i, n}\left(B_{i} \Lambda_{n}^{i-1} u_{n}-B_{i} p\right)\right\|^{2} \\
& =\left\|x_{n}-p\right\|^{2}-\left\|\Lambda_{n}^{i-1} u_{n}-\Lambda_{n}^{k} u_{n}\right\|^{2}-\lambda_{i, n}^{2}\left\|B_{i} \Lambda_{n}^{i-1} u_{n}-B_{i} p\right\|^{2} \\
& \quad+2 \lambda_{i, n}\left\langle\Lambda_{n}^{i-1} u_{n}-\Lambda_{n}^{i} u_{n}, B_{i} \Lambda_{n}^{i-1} u_{n}-B_{i} p\right\rangle \\
& \leq\left\|x_{n}-p\right\|^{2}-\left\|\Lambda_{n}^{i-1} u_{n}-\Lambda_{n}^{i} u_{n}\right\|^{2}+2 \lambda_{i, n}\left\|\Lambda_{n}^{i-1} u_{n}-\Lambda_{n}^{i} u_{n}\right\|\left\|B_{i} \Lambda_{n}^{i-1} u_{n}-B_{i} p\right\| .
\end{aligned}
$$

Combining (3.15) and (3.22), we conclude

$$
\begin{aligned}
\left\|y_{n}-p\right\|^{2} & \leq \beta_{n}\left\|x_{n}-p\right\|^{2}+\left(1-\beta_{n}\right)\left\|v_{n}-p\right\|^{2}-\frac{\beta_{n}}{1-\beta_{n}}\left\|y_{n}-x_{n}\right\|^{2} \\
& \leq \beta_{n}\left\|x_{n}-p\right\|^{2}+\left(1-\beta_{n}\right)\left\|v_{n}-p\right\|^{2}
\end{aligned}
$$




$$
\begin{aligned}
\leq & \beta_{n}\left\|x_{n}-p\right\|^{2}+\left(1-\beta_{n}\right)\left\|\Lambda_{n}^{i} u_{n}-p\right\|^{2} \\
\leq & \beta_{n}\left\|x_{n}-p\right\|^{2}+\left(1-\beta_{n}\right)\left[\left\|x_{n}-p\right\|^{2}-\left\|\Lambda_{n}^{i-1} u_{n}-\Lambda_{n}^{i} u_{n}\right\|^{2}\right. \\
& \left.+2 \lambda_{i, n}\left\|\Lambda_{n}^{i-1} u_{n}-\Lambda_{n}^{i} u_{n}\right\|\left\|B_{i} \Lambda_{n}^{i-1} u_{n}-B_{i} p\right\|\right] \\
\leq & \left\|x_{n}-p\right\|^{2}-\left(1-\beta_{n}\right)\left\|\Lambda_{n}^{i-1} u_{n}-\Lambda_{n}^{i} u_{n}\right\|^{2} \\
& +2 \lambda_{i, n}\left\|\Lambda_{n}^{i-1} u_{n}-\Lambda_{n}^{i} u_{n}\right\|\left\|B_{i} \Lambda_{n}^{i-1} u_{n}-B_{i} p\right\|,
\end{aligned}
$$

which yields

$$
\begin{aligned}
& \left(1-\beta_{n}\right)\left\|\Lambda_{n}^{i-1} u_{n}-\Lambda_{n}^{i} u_{n}\right\|^{2} \\
& \quad \leq\left\|x_{n}-p\right\|^{2}-\left\|y_{n}-p\right\|^{2}+2 \lambda_{i, n}\left\|\Lambda_{n}^{i-1} u_{n}-\Lambda_{n}^{i} u_{n}\right\|\left\|B_{i} \Lambda_{n}^{i-1} u_{n}-B_{i} p\right\| \\
& \quad \leq\left\|x_{n}-y_{n}\right\|\left(\left\|x_{n}-p\right\|+\left\|y_{n}-p\right\|\right)+2 \lambda_{i, n}\left\|\Lambda_{n}^{i-1} u_{n}-\Lambda_{n}^{i} u_{n}\right\|\left\|B_{i} \Lambda_{n}^{i-1} u_{n}-B_{i} p\right\| .
\end{aligned}
$$

Since $\left\{\beta_{n}\right\} \subset[a, b] \subset(0,1),\left\{\lambda_{i, n}\right\} \subset\left[a_{i}, b_{i}\right] \subset\left(0,2 \eta_{i}\right), i=1,2, \ldots, N$, and $\left\{u_{n}\right\},\left\{x_{n}\right\}$, and $\left\{y_{n}\right\}$ are bounded sequences, we deduce from (3.20) and $\left\|x_{n}-y_{n}\right\| \rightarrow 0$ that

$$
\lim _{n \rightarrow \infty}\left\|\Lambda_{n}^{i-1} u_{n}-\Lambda_{n}^{i} u_{n}\right\|=0, \quad \forall i \in\{1,2, \ldots, N\}
$$

Also, combining (3.3), (3.15), and (3.21), we deduce

$$
\begin{aligned}
\left\|y_{n}-p\right\|^{2} \leq & \beta_{n}\left\|x_{n}-p\right\|^{2}+\left(1-\beta_{n}\right)\left\|v_{n}-p\right\|^{2}-\frac{\beta_{n}}{1-\beta_{n}}\left\|y_{n}-x_{n}\right\|^{2} \\
\leq & \beta_{n}\left\|x_{n}-p\right\|^{2}+\left(1-\beta_{n}\right)\left\|v_{n}-p\right\|^{2} \\
\leq & \beta_{n}\left\|x_{n}-p\right\|^{2}+\left(1-\beta_{n}\right)\left\|u_{n}-p\right\|^{2} \\
\leq & \beta_{n}\left\|x_{n}-p\right\|^{2}+\left(1-\beta_{n}\right)\left\|\Delta_{n}^{k} x_{n}-p\right\|^{2} \\
\leq & \beta_{n}\left\|x_{n}-p\right\|^{2}+\left(1-\beta_{n}\right)\left[\left\|x_{n}-p\right\|^{2}-\left\|\Delta_{n}^{k-1} x_{n}-\Delta_{n}^{k} x_{n}\right\|^{2}\right. \\
& \left.+2 r_{k, n}\left\|\Delta_{n}^{k-1} x_{n}-\Delta_{n}^{k} x_{n}\right\|\left\|A_{k} \Delta_{n}^{k-1} x_{n}-A_{k} p\right\|\right] \\
\leq & \left\|x_{n}-p\right\|^{2}-\left(1-\beta_{n}\right)\left\|\Delta_{n}^{k-1} x_{n}-\Delta_{n}^{k} x_{n}\right\|^{2} \\
& +2 r_{k, n}\left\|\Delta_{n}^{k-1} x_{n}-\Delta_{n}^{k} x_{n}\right\|\left\|A_{k} \Delta_{n}^{k-1} x_{n}-A_{k} p\right\|,
\end{aligned}
$$

which yields

$$
\begin{aligned}
& \left(1-\beta_{n}\right)\left\|\Delta_{n}^{k-1} x_{n}-\Delta_{n}^{k} x_{n}\right\|^{2} \\
& \quad \leq\left\|x_{n}-p\right\|^{2}-\left\|y_{n}-p\right\|^{2}+2 r_{k, n}\left\|\Delta_{n}^{k-1} x_{n}-\Delta_{n}^{k} x_{n}\right\|\left\|A_{k} \Delta_{n}^{k-1} x_{n}-A_{k} p\right\| \\
& \quad \leq\left\|x_{n}-y_{n}\right\|\left(\left\|x_{n}-p\right\|+\left\|y_{n}-p\right\|\right)+2 r_{k, n}\left\|\Delta_{n}^{k-1} x_{n}-\Delta_{n}^{k} x_{n}\right\|\left\|A_{k} \Delta_{n}^{k-1} x_{n}-A_{k} p\right\| .
\end{aligned}
$$

Since $\left\{\beta_{n}\right\} \subset[a, b] \subset(0,1),\left\{r_{k, n}\right\} \subset\left[c_{k}, d_{k}\right] \subset\left(0,2 \mu_{k}\right)$ for $k=1,2, \ldots, M$, and $\left\{x_{n}\right\},\left\{y_{n}\right\}$ are bounded sequences, we deduce from (3.20) and $\left\|x_{n}-y_{n}\right\| \rightarrow 0$ that

$$
\lim _{n \rightarrow \infty}\left\|\Delta_{n}^{k-1} x_{n}-\Delta_{n}^{k} x_{n}\right\|=0, \quad \forall k \in\{1,2, \ldots, M\}
$$


Hence from (3.23) and (3.24), we get

$$
\begin{aligned}
\left\|x_{n}-u_{n}\right\| & =\left\|\Delta_{n}^{0} x_{n}-\Delta_{n}^{M} x_{n}\right\| \\
& \leq\left\|\Delta_{n}^{0} x_{n}-\Delta_{n}^{1} x_{n}\right\|+\left\|\Delta_{n}^{1} x_{n}-\Delta_{n}^{2} x_{n}\right\|+\cdots+\left\|\Delta_{n}^{M-1} x_{n}-\Delta_{n}^{M} x_{n}\right\| \\
& \rightarrow 0 \quad \text { as } n \rightarrow \infty
\end{aligned}
$$

and

$$
\begin{aligned}
\left\|u_{n}-v_{n}\right\| & =\left\|\Lambda_{n}^{0} u_{n}-\Lambda_{n}^{N} u_{n}\right\| \\
& \leq\left\|\Lambda_{n}^{0} u_{n}-\Lambda_{n}^{1} u_{n}\right\|+\left\|\Lambda_{n}^{1} u_{n}-\Lambda_{n}^{2} u_{n}\right\|+\cdots+\left\|\Lambda_{n}^{N-1} u_{n}-\Lambda_{n}^{N} u_{n}\right\| \\
& \rightarrow 0 \quad \text { as } n \rightarrow \infty,
\end{aligned}
$$

respectively. Thus, from (3.25) and (3.26), we obtain

$$
\left\|x_{n}-v_{n}\right\| \leq\left\|x_{n}-u_{n}\right\|+\left\|u_{n}-v_{n}\right\| \rightarrow 0 \quad \text { as } n \rightarrow \infty
$$

On the other hand, for simplicity, we write $\tilde{p}=P_{C}\left(I-v_{2} F_{2}\right) p, \tilde{v}_{n}=P_{C}\left(I-v_{2} F_{2}\right) v_{n}$, and $k_{n}=G v_{n}=P_{C}\left(I-v_{1} F_{1}\right) \tilde{v}_{n}$ for all $n \geq 1$. Then

$$
p=G p=P_{C}\left(I-v_{1} F_{1}\right) \tilde{p}=P_{C}\left(I-v_{1} F_{1}\right) P_{C}\left(I-v_{2} F_{2}\right) p .
$$

We now show that $\lim _{n \rightarrow \infty}\left\|G v_{n}-v_{n}\right\|=0$, i.e., $\lim _{n \rightarrow \infty}\left\|k_{n}-v_{n}\right\|=0$. As a matter of fact, for $p \in \Omega$, it follows from (3.4), (3.5), and (3.15) that

$$
\begin{aligned}
\left\|y_{n}-p\right\|^{2} \leq & \beta_{n}\left\|x_{n}-p\right\|^{2}+\left(1-\beta_{n}\right)\left\|G v_{n}-p\right\|^{2}-\frac{\beta_{n}}{1-\beta_{n}}\left\|y_{n}-x_{n}\right\|^{2} \\
\leq & \beta_{n}\left\|x_{n}-p\right\|^{2}+\left(1-\beta_{n}\right)\left\|G v_{n}-p\right\|^{2} \\
= & \beta_{n}\left\|x_{n}-p\right\|^{2}+\left(1-\beta_{n}\right)\left\|k_{n}-p\right\|^{2} \\
\leq & \beta_{n}\left\|x_{n}-p\right\|^{2}+\left(1-\beta_{n}\right)\left[\left\|\tilde{v}_{n}-\tilde{p}\right\|^{2}+v_{1}\left(v_{1}-2 \zeta_{1}\right)\left\|F_{1} \tilde{v}_{n}-F_{1} \tilde{p}\right\|^{2}\right] \\
\leq & \beta_{n}\left\|x_{n}-p\right\|^{2}+\left(1-\beta_{n}\right)\left[\left\|v_{n}-p\right\|^{2}+v_{2}\left(v_{2}-2 \zeta_{2}\right)\left\|F_{2} v_{n}-F_{2} p\right\|^{2}\right. \\
& \left.+v_{1}\left(v_{1}-2 \zeta_{1}\right)\left\|F_{1} \tilde{v}_{n}-F_{1} \tilde{p}\right\|^{2}\right] \\
\leq & \beta_{n}\left\|x_{n}-p\right\|^{2}+\left(1-\beta_{n}\right)\left[\left\|x_{n}-p\right\|^{2}+v_{2}\left(v_{2}-2 \zeta_{2}\right)\left\|F_{2} v_{n}-F_{2} p\right\|^{2}\right. \\
& \left.+v_{1}\left(v_{1}-2 \zeta_{1}\right)\left\|F_{1} \tilde{v}_{n}-F_{1} \tilde{p}\right\|^{2}\right] \\
= & \left\|x_{n}-p\right\|^{2}+\left(1-\beta_{n}\right)\left[v_{2}\left(v_{2}-2 \zeta_{2}\right)\left\|F_{2} v_{n}-F_{2} p\right\|^{2}\right. \\
& \left.+v_{1}\left(v_{1}-2 \zeta_{1}\right)\left\|F_{1} \tilde{v}_{n}-F_{1} \tilde{p}\right\|^{2}\right],
\end{aligned}
$$

which immediately yields

$$
\begin{aligned}
& \left(1-\beta_{n}\right)\left[v_{2}\left(2 \zeta_{2}-v_{2}\right)\left\|F_{2} v_{n}-F_{2} p\right\|^{2}+v_{1}\left(2 \zeta_{1}-v_{1}\right)\left\|F_{1} \tilde{v}_{n}-F_{1} \tilde{p}\right\|^{2}\right] \\
& \quad \leq\left\|x_{n}-p\right\|^{2}-\left\|y_{n}-p\right\|^{2} \\
& \quad \leq\left\|x_{n}-y_{n}\right\|\left(\left\|x_{n}-p\right\|+\left\|y_{n}-p\right\|\right) .
\end{aligned}
$$


Since $\left\|x_{n}-y_{n}\right\| \rightarrow 0,\left\{\beta_{n}\right\} \subset[a, b] \subset(0,1), v_{j} \in\left(0,2 \zeta_{j}\right), j=1,2$, and $\left\{x_{n}\right\},\left\{y_{n}\right\}$ are bounded sequences, we have

$$
\lim _{n \rightarrow \infty}\left\|F_{2} v_{n}-F_{2} p\right\|=0 \quad \text { and } \quad \lim _{n \rightarrow \infty}\left\|F_{1} \tilde{v}_{n}-F_{1} \tilde{p}\right\|=0 .
$$

Also, in terms of the firm nonexpansivity of $P_{C}$ and the $\zeta_{j}$-inverse-strong monotonicity of $F_{j}$ for $j=1,2$, we obtain from $v_{j} \in\left(0,2 \zeta_{j}\right), j=1,2$,

$$
\begin{aligned}
\left\|\tilde{v}_{n}-\tilde{p}\right\|^{2}= & \left\|P_{C}\left(I-v_{2} F_{2}\right) v_{n}-P_{C}\left(I-v_{2} F_{2}\right) p\right\|^{2} \\
\leq & \left\langle\left(I-v_{2} F_{2}\right) v_{n}-\left(I-v_{2} F_{2}\right) p, \tilde{v}_{n}-\tilde{p}\right\rangle \\
= & \frac{1}{2}\left[\left\|\left(I-v_{2} F_{2}\right) v_{n}-\left(I-v_{2} F_{2}\right) p\right\|^{2}+\left\|\tilde{v}_{n}-\tilde{p}\right\|^{2}\right. \\
& \left.-\left\|\left(I-v_{2} F_{2}\right) v_{n}-\left(I-v_{2} F_{2}\right) p-\left(\tilde{v}_{n}-\tilde{p}\right)\right\|^{2}\right] \\
\leq & \frac{1}{2}\left[\left\|v_{n}-p\right\|^{2}+\left\|\tilde{v}_{n}-\tilde{p}\right\|^{2}-\left\|\left(v_{n}-\tilde{v}_{n}\right)-v_{2}\left(F_{2} v_{n}-F_{2} p\right)-(p-\tilde{p})\right\|^{2}\right] \\
= & \frac{1}{2}\left[\left\|v_{n}-p\right\|^{2}+\left\|\tilde{v}_{n}-\tilde{p}\right\|^{2}-\left\|\left(v_{n}-\tilde{v}_{n}\right)-(p-\tilde{p})\right\|^{2}\right. \\
& \left.+2 v_{2}\left(\left(v_{n}-\tilde{v}_{n}\right)-(p-\tilde{p}), F_{2} v_{n}-F_{2} p\right\rangle-v_{2}^{2}\left\|F_{2} v_{n}-F_{2} p\right\|^{2}\right]
\end{aligned}
$$

and

$$
\begin{aligned}
\left\|k_{n}-p\right\|^{2}= & \left\|P_{C}\left(I-v_{1} F_{1}\right) \tilde{v}_{n}-P_{C}\left(I-v_{1} F_{1}\right) \tilde{p}\right\|^{2} \\
\leq & \left\langle\left(I-v_{1} F_{1}\right) \tilde{v}_{n}-\left(I-v_{1} F_{1}\right) \tilde{p}, k_{n}-p\right\rangle \\
= & \frac{1}{2}\left[\left\|\left(I-v_{1} F_{1}\right) \tilde{v}_{n}-\left(I-v_{1} F_{1}\right) \tilde{p}\right\|^{2}+\left\|k_{n}-p\right\|^{2}\right. \\
& \left.-\left\|\left(I-v_{1} F_{1}\right) \tilde{v}_{n}-\left(I-v_{1} F_{1}\right) \tilde{p}-\left(k_{n}-p\right)\right\|^{2}\right] \\
\leq & \frac{1}{2}\left[\left\|\tilde{v}_{n}-\tilde{p}\right\|^{2}+\left\|k_{n}-p\right\|^{2}-\left\|\left(\tilde{v}_{n}-k_{n}\right)+(p-\tilde{p})\right\|^{2}\right. \\
& \left.+2 v_{1}\left\langle F_{1} \tilde{v}_{n}-F_{1} \tilde{p},\left(\tilde{v}_{n}-k_{n}\right)+(p-\tilde{p})\right\rangle-v_{1}^{2}\left\|F_{1} \tilde{v}_{n}-F_{1} \tilde{p}\right\|^{2}\right] \\
\leq & \frac{1}{2}\left[\left\|v_{n}-p\right\|^{2}+\left\|w_{n}-p\right\|^{2}-\left\|\left(\tilde{v}_{n}-k_{n}\right)+(p-\tilde{p})\right\|^{2}\right. \\
& \left.+2 v_{1}\left(F_{1} \tilde{v}_{n}-F_{1} \tilde{p},\left(\tilde{v}_{n}-k_{n}\right)+(p-\tilde{p})\right\rangle\right] .
\end{aligned}
$$

Thus, we have

$$
\begin{aligned}
\left\|\tilde{v}_{n}-\tilde{p}\right\|^{2} \leq & \left\|v_{n}-p\right\|^{2}-\left\|\left(v_{n}-\tilde{v}_{n}\right)-(p-\tilde{p})\right\|^{2} \\
& +2 v_{2}\left(\left(v_{n}-\tilde{v}_{n}\right)-(p-\tilde{p}), F_{2} v_{n}-F_{2} p\right\rangle-v_{2}^{2}\left\|F_{2} v_{n}-F_{2} p\right\|^{2}
\end{aligned}
$$

and

$$
\begin{aligned}
\left\|k_{n}-p\right\|^{2} \leq & \left\|v_{n}-p\right\|^{2}-\left\|\left(\tilde{v}_{n}-k_{n}\right)+(p-\tilde{p})\right\|^{2} \\
& +2 v_{1}\left\|F_{1} \tilde{v}_{n}-F_{1} \tilde{p}\right\|\left\|\left(\tilde{v}_{n}-k_{n}\right)+(p-\tilde{p})\right\| .
\end{aligned}
$$


Consequently, from (3.4), (3.28), and (3.30), it follows that

$$
\begin{aligned}
\left\|y_{n}-p\right\|^{2} \leq & \beta_{n}\left\|x_{n}-p\right\|^{2}+\left(1-\beta_{n}\right)\left[\left\|\tilde{v}_{n}-\tilde{p}\right\|^{2}+v_{1}\left(v_{1}-2 \zeta_{1}\right)\left\|F_{1} \tilde{v}_{n}-F_{1} \tilde{p}\right\|^{2}\right] \\
\leq & \beta_{n}\left\|x_{n}-p\right\|^{2}+\left(1-\beta_{n}\right)\left\|\tilde{v}_{n}-\tilde{p}\right\|^{2} \\
\leq & \beta_{n}\left\|x_{n}-p\right\|^{2}+\left(1-\beta_{n}\right)\left[\left\|v_{n}-p\right\|^{2}-\left\|\left(v_{n}-\tilde{v}_{n}\right)-(p-\tilde{p})\right\|^{2}\right. \\
& \left.+2 v_{2}\left\langle\left(v_{n}-\tilde{v}_{n}\right)-(p-\tilde{p}), F_{2} v_{n}-F_{2} p\right\rangle-v_{2}^{2}\left\|F_{2} v_{n}-F_{2} p\right\|^{2}\right] \\
\leq & \beta_{n}\left\|x_{n}-p\right\|^{2}+\left(1-\beta_{n}\right)\left[\left\|x_{n}-p\right\|^{2}-\left\|\left(v_{n}-\tilde{v}_{n}\right)-(p-\tilde{p})\right\|^{2}\right. \\
& \left.+2 v_{2}\left\|\left(v_{n}-\tilde{v}_{n}\right)-(p-\tilde{p})\right\|\left\|F_{2} v_{n}-F_{2} p\right\|\right] \\
\leq & \left\|x_{n}-p\right\|^{2}-\left(1-\beta_{n}\right)\left\|\left(v_{n}-\tilde{v}_{n}\right)-(p-\tilde{p})\right\|^{2} \\
& +2 v_{2}\left\|\left(v_{n}-\tilde{v}_{n}\right)-(p-\tilde{p})\right\|\left\|F_{2} v_{n}-F_{2} p\right\|,
\end{aligned}
$$

which hence leads to

$$
\begin{aligned}
& \left(1-\beta_{n}\right)\left\|\left(v_{n}-\tilde{v}_{n}\right)-(p-\tilde{p})\right\|^{2} \\
& \quad \leq\left\|x_{n}-p\right\|^{2}-\left\|y_{n}-p\right\|^{2}+2 v_{2}\left\|\left(v_{n}-\tilde{v}_{n}\right)-(p-\tilde{p})\right\|\left\|F_{2} v_{n}-F_{2} p\right\| \\
& \quad \leq\left\|x_{n}-y_{n}\right\|\left(\left\|x_{n}-p\right\|+\left\|y_{n}-p\right\|\right)+2 v_{2}\left\|\left(v_{n}-\tilde{v}_{n}\right)-(p-\tilde{p})\right\|\left\|F_{2} v_{n}-F_{2} p\right\| .
\end{aligned}
$$

Since $\left\|x_{n}-y_{n}\right\| \rightarrow 0,\left\{\beta_{n}\right\} \subset[a, b] \subset(0,1), v_{2} \in\left(0,2 \zeta_{2}\right)$, and $\left\{x_{n}\right\},\left\{y_{n}\right\},\left\{v_{n}\right\},\left\{\tilde{v}_{n}\right\}$ are bounded sequences, we obtain from (3.29)

$$
\lim _{n \rightarrow \infty}\left\|\left(v_{n}-\tilde{v}_{n}\right)-(p-\tilde{p})\right\|=0 .
$$

Furthermore, from (3.4), (3.28), and (3.31), it follows that

$$
\begin{aligned}
\left\|y_{n}-p\right\|^{2} \leq & \beta_{n}\left\|x_{n}-p\right\|^{2}+\left(1-\beta_{n}\right)\left\|k_{n}-p\right\|^{2} \\
\leq & \beta_{n}\left\|x_{n}-p\right\|^{2}+\left(1-\beta_{n}\right)\left[\left\|v_{n}-p\right\|^{2}-\left\|\left(\tilde{v}_{n}-k_{n}\right)+(p-\tilde{p})\right\|^{2}\right. \\
& \left.+2 v_{1}\left\|F_{1} \tilde{v}_{n}-F_{1} \tilde{p}\right\|\left\|\left(\tilde{v}_{n}-k_{n}\right)+(p-\tilde{p})\right\|\right] \\
\leq & \beta_{n}\left\|x_{n}-p\right\|^{2}+\left(1-\beta_{n}\right)\left[\left\|x_{n}-p\right\|^{2}-\left\|\left(\tilde{v}_{n}-k_{n}\right)+(p-\tilde{p})\right\|^{2}\right. \\
& \left.+2 v_{1}\left\|F_{1} \tilde{v}_{n}-F_{1} \tilde{p}\right\|\left\|\left(\tilde{v}_{n}-k_{n}\right)+(p-\tilde{p})\right\|\right] \\
= & \left\|x_{n}-p\right\|^{2}-\left(1-\beta_{n}\right)\left\|\left(\tilde{v}_{n}-k_{n}\right)+(p-\tilde{p})\right\|^{2} \\
& +2 v_{1}\left\|F_{1} \tilde{v}_{n}-F_{1} \tilde{p}\right\|\left\|\left(\tilde{v}_{n}-k_{n}\right)+(p-\tilde{p})\right\|,
\end{aligned}
$$

which hence yields

$$
\begin{aligned}
& \left(1-\beta_{n}\right)\left\|\left(\tilde{v}_{n}-k_{n}\right)+(p-\tilde{p})\right\|^{2} \\
& \quad \leq\left\|x_{n}-p\right\|^{2}-\left\|y_{n}-p\right\|^{2}+2 v_{1}\left\|F_{1} \tilde{v}_{n}-F_{1} \tilde{p}\right\|\left\|\left(\tilde{v}_{n}-k_{n}\right)+(p-\tilde{p})\right\| \\
& \quad \leq\left\|x_{n}-y_{n}\right\|\left(\left\|x_{n}-p\right\|+\left\|y_{n}-p\right\|\right)+2 v_{1}\left\|F_{1} \tilde{v}_{n}-F_{1} \tilde{p}\right\|\left\|\left(\tilde{v}_{n}-k_{n}\right)+(p-\tilde{p})\right\| .
\end{aligned}
$$


Since $\left\|x_{n}-y_{n}\right\| \rightarrow 0,\left\{\beta_{n}\right\} \subset[a, b] \subset(0,1), v_{1} \in\left(0,2 \zeta_{1}\right)$, and $\left\{x_{n}\right\},\left\{y_{n}\right\},\left\{k_{n}\right\},\left\{\tilde{v}_{n}\right\}$ are bounded sequences, we obtain from (3.29)

$$
\lim _{n \rightarrow \infty}\left\|\left(\tilde{v}_{n}-k_{n}\right)+(p-\tilde{p})\right\|=0
$$

Note that

$$
\left\|v_{n}-k_{n}\right\| \leq\left\|\left(v_{n}-\tilde{v}_{n}\right)-(p-\tilde{p})\right\|+\left\|\left(\tilde{v}_{n}-k_{n}\right)+(p-\tilde{p})\right\| .
$$

Hence from (3.32) and (3.33), we get

$$
\lim _{n \rightarrow \infty}\left\|v_{n}-G v_{n}\right\|=\lim _{n \rightarrow \infty}\left\|v_{n}-k_{n}\right\|=0
$$

Also, observe that

$$
y_{n}-x_{n}=\gamma_{n}\left(G v_{n}-x_{n}\right)+\delta_{n}\left(T G v_{n}-x_{n}\right), \quad \forall n \geq 0 .
$$

Hence we find that

$$
\begin{aligned}
\delta_{n}\left\|T G v_{n}-v_{n}\right\| & \leq \delta_{n}\left\|T G v_{n}-x_{n}\right\|+\delta_{n}\left\|x_{n}-v_{n}\right\| \\
& =\left\|y_{n}-x_{n}-\gamma_{n}\left(G v_{n}-x_{n}\right)\right\|+\delta_{n}\left\|x_{n}-v_{n}\right\| \\
& \leq\left\|y_{n}-x_{n}\right\|+\gamma_{n}\left\|G v_{n}-x_{n}\right\|+\delta_{n}\left\|x_{n}-v_{n}\right\| \\
& \leq\left\|y_{n}-x_{n}\right\|+\gamma_{n}\left\|G v_{n}-v_{n}\right\|+\gamma_{n}\left\|v_{n}-x_{n}\right\|+\delta_{n}\left\|x_{n}-v_{n}\right\| \\
& =\left\|y_{n}-x_{n}\right\|+\gamma_{n}\left\|G v_{n}-v_{n}\right\|+\left(\gamma_{n}+\delta_{n}\right)\left\|x_{n}-v_{n}\right\| \\
& \leq\left\|y_{n}-x_{n}\right\|+\left\|G v_{n}-v_{n}\right\|+\left\|x_{n}-v_{n}\right\| .
\end{aligned}
$$

So, from $\liminf \operatorname{in}_{n \rightarrow \infty} \delta_{n}>0$, (3.17), (3.27), and (3.34), it follows that

$$
\lim _{n \rightarrow \infty}\left\|T G v_{n}-v_{n}\right\|=0
$$

In addition, noticing that

$$
\begin{aligned}
\left\|T v_{n}-v_{n}\right\| & \leq\left\|T v_{n}-T G v_{n}\right\|+\left\|T G v_{n}-v_{n}\right\| \\
& \leq\left\|v_{n}-G v_{n}\right\|+\left\|T G v_{n}-v_{n}\right\|,
\end{aligned}
$$

we know from (3.34) and (3.35) that

$$
\lim _{n \rightarrow \infty}\left\|T v_{n}-v_{n}\right\|=0
$$

Step 4. We prove that $\omega_{w}\left(x_{n}\right) \subset \Omega$.

Indeed, since $H$ is reflexive and $\left\{x_{n}\right\}$ is bounded, there exists at least a weak convergence subsequence of $\left\{x_{n}\right\}$. Hence it is well known that $\omega_{w}\left(x_{n}\right) \neq \emptyset$. Now, take an arbitrary $w \in$ $\omega_{w}\left(x_{n}\right)$. Then there exists a subsequence $\left\{x_{n_{i}}\right\}$ of $\left\{x_{n}\right\}$ such that $x_{n_{i}} \rightarrow w$. From (3.23)-(3.25), and (3.27), we have $u_{n_{i}} \rightarrow w, v_{n_{i}} \rightarrow w, \Lambda_{n_{i}}^{m} u_{n_{i}} \rightarrow w$, and $\Delta_{n_{i}}^{k} x_{n_{i}} \rightarrow w$, where $m \in\{1,2, \ldots, N\}$ 
and $k \in\{1,2, \ldots, M\}$. Utilizing Lemma 2.1(ii), we deduce from $v_{n_{i}} \rightarrow w$ and (3.36) that $w \in \operatorname{Fix}(T)$. In the meantime, utilizing Lemma 2.10, we obtain from $v_{n_{i}} \rightarrow w$ and (3.34) $w \in \operatorname{GSVI}(G)$. Next, we prove that $w \in \bigcap_{m=1}^{N} \mathrm{I}\left(B_{m}, R_{m}\right)$. As a matter of fact, since $B_{m}$ is $\eta_{m}$-inverse-strongly monotone, $B_{m}$ is a monotone and Lipschitz continuous mapping. It follows from Lemma 2.6 that $R_{m}+B_{m}$ is maximal monotone. Let $(\nu, g) \in G\left(R_{m}+B_{m}\right)$, i.e., $g-B_{m} v \in R_{m} v$. Again, since $\Lambda_{n}^{m} u_{n}=J_{R_{m}, \lambda_{m, n}}\left(I-\lambda_{m, n} B_{m}\right) \Lambda_{n}^{m-1} u_{n}, n \geq 1, m \in\{1,2, \ldots, N\}$, we have

$$
\Lambda_{n}^{m-1} u_{n}-\lambda_{m, n} B_{m} \Lambda_{n}^{m-1} u_{n} \in\left(I+\lambda_{m, n} R_{m}\right) \Lambda_{n}^{m} u_{n}
$$

that is,

$$
\frac{1}{\lambda_{m, n}}\left(\Lambda_{n}^{m-1} u_{n}-\Lambda_{n}^{m} u_{n}-\lambda_{m, n} B_{m} \Lambda_{n}^{m-1} u_{n}\right) \in R_{m} \Lambda_{n}^{m} u_{n}
$$

In terms of the monotonicity of $R_{m}$, we get

$$
\left\langle v-\Lambda_{n}^{m} u_{n}, g-B_{m} v-\frac{1}{\lambda_{m, n}}\left(\Lambda_{n}^{m-1} u_{n}-\Lambda_{n}^{m} u_{n}-\lambda_{m, n} B_{m} \Lambda_{n}^{m-1} u_{n}\right)\right\rangle \geq 0
$$

and hence

$$
\begin{aligned}
\langle v & \left.-\Lambda_{n}^{m} u_{n}, g\right\rangle \\
& \geq\left\langle v-\Lambda_{n}^{m} u_{n}, B_{m} v+\frac{1}{\lambda_{m, n}}\left(\Lambda_{n}^{m-1} u_{n}-\Lambda_{n}^{m} u_{n}-\lambda_{m, n} B_{m} \Lambda_{n}^{m-1} u_{n}\right)\right\rangle \\
& =\left\langle v-\Lambda_{n}^{m} u_{n}, B_{m} v-B_{m} \Lambda_{n}^{m} u_{n}+B_{m} \Lambda_{n}^{m} u_{n}-B_{m} \Lambda_{n}^{m-1} u_{n}+\frac{1}{\lambda_{m, n}}\left(\Lambda_{n}^{m-1} u_{n}-\Lambda_{n}^{m} u_{n}\right)\right\rangle \\
& \geq\left\langle v-\Lambda_{n}^{m} u_{n}, B_{m} \Lambda_{n}^{m} u_{n}-B_{m} \Lambda_{n}^{m-1} u_{n}\right\rangle+\left\langle v-\Lambda_{n}^{m} u_{n}, \frac{1}{\lambda_{m, n}}\left(\Lambda_{n}^{m-1} u_{n}-\Lambda_{n}^{m} u_{n}\right)\right\rangle .
\end{aligned}
$$

In particular,

$$
\begin{aligned}
\left\langle v-\Lambda_{n_{i}}^{m} u_{n_{i}}, g\right\rangle \geq & \left\langle v-\Lambda_{n_{i}}^{m} u_{n_{i}}, B_{m} \Lambda_{n_{i}}^{m} u_{n_{i}}-B_{m} \Lambda_{n_{i}}^{m-1} u_{n_{i}}\right\rangle \\
& +\left\langle v-\Lambda_{n_{i}}^{m} u_{n_{i}}, \frac{1}{\lambda_{m, n_{i}}}\left(\Lambda_{n_{i}}^{m-1} u_{n_{i}}-\Lambda_{n_{i}}^{m} u_{n_{i}}\right)\right\rangle .
\end{aligned}
$$

Since $\left\|\Lambda_{n}^{m} u_{n}-\Lambda_{n}^{m-1} u_{n}\right\| \rightarrow 0$ (due to (3.23)) and $\left\|B_{m} \Lambda_{n}^{m} u_{n}-B_{m} \Lambda_{n}^{m-1} u_{n}\right\| \rightarrow 0$ (due to the Lipschitz continuity of $\left.B_{m}\right)$, we conclude from $\Lambda_{n_{i}}^{m} u_{n_{i}} \rightarrow w$ and $\left\{\lambda_{i, n}\right\} \subset\left[a_{i}, b_{i}\right] \subset\left(0,2 \eta_{i}\right)$ that

$$
\lim _{i \rightarrow \infty}\left\langle v-\Lambda_{n_{i}}^{m} u_{n_{i}}, g\right\rangle=\langle v-w, g\rangle \geq 0 .
$$

It follows from the maximal monotonicity of $B_{m}+R_{m}$ that $0 \in\left(R_{m}+B_{m}\right) w$, i.e., $w \in$ $\mathrm{I}\left(B_{m}, R_{m}\right)$. Therefore, $w \in \bigcap_{m=1}^{N} \mathrm{I}\left(B_{m}, R_{m}\right)$. Next we prove that $w \in \bigcap_{k=1}^{M} \operatorname{GMEP}\left(\Theta_{k}, \varphi_{k}, A_{k}\right)$. Since $\Delta_{n}^{k} x_{n}=T_{r_{k, n}}^{\left(\Theta_{k}, \varphi_{k}\right)}\left(I-r_{k, n} A_{k}\right) \Delta_{n}^{k-1} x_{n}, n \geq 1, k \in\{1,2, \ldots, M\}$, we have

$$
\begin{aligned}
& \Theta_{k}\left(\Delta_{n}^{k} x_{n}, y\right)+\varphi_{k}(y)-\varphi_{k}\left(\Delta_{n}^{k} x_{n}\right)+\left\langle A_{k} \Delta_{n}^{k-1} x_{n}, y-\Delta_{n}^{k} x_{n}\right\rangle \\
& +\frac{1}{r_{k, n}}\left\langle y-\Delta_{n}^{k} x_{n}, \Delta_{n}^{k} x_{n}-\Delta_{n}^{k-1} x_{n}\right\rangle \geq 0
\end{aligned}
$$


By (A2), we have

$$
\varphi_{k}(y)-\varphi_{k}\left(\Delta_{n}^{k} x_{n}\right)+\left\langle A_{k} \Delta_{n}^{k-1} x_{n}, y-\Delta_{n}^{k} x_{n}\right\rangle+\frac{1}{r_{k, n}}\left\langle y-\Delta_{n}^{k} x_{n}, \Delta_{n}^{k} x_{n}-\Delta_{n}^{k-1} x_{n}\right\rangle \geq \Theta_{k}\left(y, \Delta_{n}^{k} x_{n}\right) .
$$

Let $z_{t}=t y+(1-t) w$ for all $t \in(0,1]$ and $y \in C$. This implies that $z_{t} \in C$. Then we have

$$
\begin{aligned}
& \left\langle z_{t}-\Delta_{n}^{k} x_{n}, A_{k} z_{t}\right\rangle \\
& \geq \varphi_{k}\left(\Delta_{n}^{k} x_{n}\right)-\varphi_{k}\left(z_{t}\right)+\left\langle z_{t}-\Delta_{n}^{k} x_{n}, A_{k} z_{t}\right\rangle-\left\langle z_{t}-\Delta_{n}^{k} x_{n}, A_{k} \Delta_{n}^{k-1} x_{n}\right\rangle \\
& \quad-\left\langle z_{t}-\Delta_{n}^{k} x_{n}, \frac{\Delta_{n}^{k} x_{n}-\Delta_{n}^{k-1} x_{n}}{r_{k, n}}\right\rangle+\Theta_{k}\left(z_{t}, \Delta_{n}^{k} x_{n}\right) \\
& =\varphi_{k}\left(\Delta_{n}^{k} x_{n}\right)-\varphi_{k}\left(z_{t}\right)+\left\langle z_{t}-\Delta_{n}^{k} x_{n}, A_{k} z_{t}-A_{k} \Delta_{n}^{k} x_{n}\right\rangle+\left\langle z_{t}-\Delta_{n}^{k} x_{n}, A_{k} \Delta_{n}^{k} x_{n}-A_{k} \Delta_{n}^{k-1} x_{n}\right\rangle \\
& \quad-\left\langle z_{t}-\Delta_{n}^{k} x_{n}, \frac{\Delta_{n}^{k} x_{n}-\Delta_{n}^{k-1} x_{n}}{r_{k, n}}\right\rangle+\Theta_{k}\left(z_{t}, \Delta_{n}^{k} x_{n}\right) .
\end{aligned}
$$

By (3.24), we have $\left\|A_{k} \Delta_{n}^{k} x_{n}-A_{k} \Delta_{n}^{k-1} x_{n}\right\| \rightarrow 0$ as $n \rightarrow \infty$. Furthermore, by the monotonicity of $A_{k}$, we obtain $\left\langle z_{t}-\Delta_{n}^{k} x_{n}, A_{k} z_{t}-A_{k} \Delta_{n}^{k} x_{n}\right\rangle \geq 0$. Then by (A4) we obtain

$$
\left\langle z_{t}-w, A_{k} z_{t}\right\rangle \geq \varphi_{k}(w)-\varphi_{k}\left(z_{t}\right)+\Theta_{k}\left(z_{t}, w\right)
$$

Utilizing (A1), (A4), and (3.26), we obtain

$$
\begin{aligned}
0 & =\Theta_{k}\left(z_{t}, z_{t}\right)+\varphi_{k}\left(z_{t}\right)-\varphi_{k}\left(z_{t}\right) \\
& \leq t \Theta_{k}\left(z_{t}, y\right)+(1-t) \Theta_{k}\left(z_{t}, w\right)+t \varphi_{k}(y)+(1-t) \varphi_{k}(w)-\varphi_{k}\left(z_{t}\right) \\
& \leq t\left[\Theta_{k}\left(z_{t}, y\right)+\varphi_{k}(y)-\varphi_{k}\left(z_{t}\right)\right]+(1-t)\left\langle z_{t}-w, A_{k} z_{t}\right\rangle \\
& =t\left[\Theta_{k}\left(z_{t}, y\right)+\varphi_{k}(y)-\varphi_{k}\left(z_{t}\right)\right]+(1-t) t\left\langle y-w, A_{k} z_{t}\right\rangle
\end{aligned}
$$

and hence

$$
0 \leq \Theta_{k}\left(z_{t}, y\right)+\varphi_{k}(y)-\varphi_{k}\left(z_{t}\right)+(1-t)\left\langle y-w, A_{k} z_{t}\right\rangle
$$

Letting $t \rightarrow 0$, we have, for each $y \in C$,

$$
0 \leq \Theta_{k}(w, y)+\varphi_{k}(y)-\varphi_{k}(w)+\left\langle y-w, A_{k} w\right\rangle
$$

This implies that $w \in \operatorname{GMEP}\left(\Theta_{k}, \varphi_{k}, A_{k}\right)$, and hence, $w \in \bigcap_{k=1}^{M} \operatorname{GMEP}\left(\Theta_{k}, \varphi_{k}, A_{k}\right)$. Thus, $w \in \Omega=\bigcap_{n=1}^{\infty} \operatorname{Fix}\left(T_{n}\right) \cap \bigcap_{k=1}^{M} \operatorname{GMEP}\left(\Theta_{k}, \varphi_{k}, A_{k}\right) \cap \bigcap_{m=1}^{N} \mathrm{I}\left(B_{m}, R_{m}\right)$. Consequently, $w \in$ $\bigcap_{k=1}^{M} \operatorname{GMEP}\left(\Theta_{k}, \varphi_{k}, A_{k}\right) \cap \bigcap_{m=1}^{N} \mathrm{I}\left(B_{m}, R_{m}\right) \cap \operatorname{GSVI}(G) \cap \operatorname{Fix}(T)=: \Omega$. This shows that $\omega_{w}\left(x_{n}\right) \subset \Omega$.

Step 5. We prove that $\omega_{w}\left(x_{n}\right) \subset \Xi$.

Indeed, take an arbitrary $w \in \omega_{w}\left(x_{n}\right)$. Then there exists a subsequence $\left\{x_{n_{i}}\right\}$ of $\left\{x_{n}\right\}$ such that $x_{n_{i}} \rightarrow w$. Utilizing (3.16), we obtain, for all $p \in \Omega$,

$$
\begin{aligned}
& \left\|x_{n+1}-p\right\|^{2} \\
& \quad \leq\left(1-\lambda_{n} \frac{\tau^{2}-\gamma^{2}}{\tau}\right)\left\|x_{n}-p\right\|^{2}-\frac{\beta_{n}\left(1-\lambda_{n} \tau\right)}{1-\beta_{n}}\left\|y_{n}-x_{n}\right\|^{2}
\end{aligned}
$$




$$
\begin{aligned}
& +2 \lambda_{n} \alpha_{n}\left\langle(\gamma V p-\mu F p), x_{n+1}-p\right\rangle+2 \lambda_{n}\left(1-\alpha_{n}\right)\left\langle(\gamma S p-\mu F p), x_{n+1}-p\right\rangle \\
\leq & \left\|x_{n}-p\right\|^{2}+2 \lambda_{n} \alpha_{n}\left\langle(\gamma V-\mu F) p, x_{n+1}-p\right\rangle+2 \lambda_{n}\left(1-\alpha_{n}\right)\left\langle(\gamma S p-\mu F p), x_{n+1}-p\right\rangle,
\end{aligned}
$$

which implies that

$$
\begin{aligned}
& \left\langle(\mu F-\gamma S) p, x_{n}-p\right\rangle \\
& \leq\left\langle(\mu F-\gamma S) p, x_{n}-x_{n+1}\right\rangle+\left\langle(\mu F-\gamma S) p, x_{n+1}-p\right\rangle \\
& \leq\|(\mu F-\gamma S) p\|\left\|x_{n}-x_{n+1}\right\|+\frac{\left\|x_{n}-p\right\|^{2}-\left\|x_{n+1}-p\right\|^{2}}{2 \lambda_{n}\left(1-\alpha_{n}\right)} \\
& \quad+\frac{\alpha_{n}}{1-\alpha_{n}}\left\langle(\gamma V-\mu F) p, x_{n+1}-p\right\rangle \\
& \leq\|(\mu F-\gamma S) p\|\left\|x_{n}-x_{n+1}\right\|+\frac{\left\|x_{n}-x_{n+1}\right\|\left(\left\|x_{n}-p\right\|+\left\|x_{n+1}-p\right\|\right)}{2 \lambda_{n}\left(1-\alpha_{n}\right)} \\
& \quad+\frac{\alpha_{n}}{1-\alpha_{n}}\|(\gamma V-\mu F) p\|\left\|x_{n+1}-p\right\| .
\end{aligned}
$$

Since $\alpha_{n} \rightarrow 0,\left\|x_{n}-x_{n+1}\right\| \rightarrow 0$ and

$$
\lim _{n \rightarrow \infty} \frac{\left\|x_{n}-x_{n+1}\right\|}{\lambda_{n}}=\lim _{n \rightarrow \infty} \frac{\left\|x_{n}-x_{n+1}\right\|}{\alpha_{n}} \cdot \frac{\alpha_{n}}{\lambda_{n}}=0,
$$

from (3.39), we conclude that

$$
\begin{aligned}
\langle(\mu F-\gamma S) p, w-p\rangle & =\lim _{i \rightarrow \infty}\left\langle(\mu F-\gamma S) p, x_{n_{i}}-p\right\rangle \\
& \leq \limsup _{n \rightarrow \infty}\left\langle(\mu F-\gamma S) p, x_{n}-p\right\rangle \\
& \leq 0, \quad \forall p \in \Omega,
\end{aligned}
$$

that is,

$$
\langle(\mu F-\gamma S) p, w-p\rangle \leq 0, \quad \forall p \in \Omega
$$

Since $\mu F-\gamma S$ is $(\mu \eta-\gamma)$-strongly monotone and $(\mu \kappa+\gamma)$-Lipschitz continuous, by Minty's lemma [29] we know that (3.40) is equivalent to the VIP

$$
\langle(\mu F-\gamma S) w, p-w\rangle \geq 0, \quad \forall p \in \Omega
$$

This shows that $w \in \operatorname{VI}(\Omega, \mu F-\gamma S)$. Taking into account $\left\{x^{*}\right\}=\operatorname{VI}(\Omega, \mu F-\gamma S)$, we know that $w=x^{*}$. Thus, $\omega_{w}\left(x_{n}\right)=\left\{x^{*}\right\}$; that is, $x_{n} \rightarrow x^{*}$.

Next we prove that $\lim _{n \rightarrow \infty}\left\|x_{n}-x^{*}\right\|=0$. As a matter of fact, utilizing (3.16) with $p=x^{*}$, we get

$$
\begin{aligned}
& \left\|x_{n+1}-x^{*}\right\|^{2} \\
& \quad \leq\left(1-\lambda_{n} \frac{\tau^{2}-\gamma^{2}}{\tau}\right)\left\|x_{n}-x^{*}\right\|^{2}-\frac{\beta_{n}\left(1-\lambda_{n} \tau\right)}{1-\beta_{n}}\left\|y_{n}-x_{n}\right\|^{2}
\end{aligned}
$$




$$
\begin{aligned}
& +2 \lambda_{n} \alpha_{n}\left\langle\left(\gamma V x^{*}-\mu F x^{*}\right), x_{n+1}-x^{*}\right\rangle+2 \lambda_{n}\left(1-\alpha_{n}\right)\left\langle\left(\gamma S x^{*}-\mu F x^{*}\right), x_{n+1}-x^{*}\right\rangle \\
\leq & \left(1-\lambda_{n} \frac{\tau^{2}-\gamma^{2}}{\tau}\right)\left\|x_{n}-x^{*}\right\|^{2}+2 \lambda_{n} \alpha_{n}\left\|(\gamma V-\mu F) x^{*}\right\|\left\|x_{n+1}-x^{*}\right\| \\
& +2 \lambda_{n}\left(1-\alpha_{n}\right)\left\langle\left(\gamma S x^{*}-\mu F x^{*}\right), x_{n+1}-x^{*}\right\rangle \\
= & \left(1-\lambda_{n} \frac{\tau^{2}-\gamma^{2}}{\tau}\right)\left\|x_{n}-x^{*}\right\|^{2}+\lambda_{n} \frac{\tau^{2}-\gamma^{2}}{\tau} \cdot \frac{2 \tau}{\tau^{2}-\gamma^{2}}\left[\alpha_{n}\left\|(\gamma V-\mu F) x^{*}\right\|\left\|x_{n+1}-x^{*}\right\|\right. \\
& \left.+\left(1-\alpha_{n}\right)\left\langle\left(\gamma S x^{*}-\mu F x^{*}\right), x_{n+1}-x^{*}\right\rangle\right] .
\end{aligned}
$$

Since $\sum_{n=0}^{\infty} \lambda_{n}=\infty$ and $\lim _{n \rightarrow \infty}\left\langle\left(\gamma S x^{*}-\mu F x^{*}\right), x^{*}-x_{n+1}\right\rangle=0$ (due to $x_{n} \rightarrow x^{*}$ ), we deduce that $\sum_{n=0}^{\infty} \lambda_{n} \frac{\tau^{2}-\gamma^{2}}{\tau}=\infty$, and

$$
\lim _{n \rightarrow \infty} \frac{2 \tau}{\tau^{2}-\gamma^{2}}\left[\alpha_{n}\left\|(\gamma V-\mu F) x^{*}\right\|\left\|x_{n+1}-x^{*}\right\|+\left(1-\alpha_{n}\right)\left\langle\left(\gamma S x^{*}-\mu F x^{*}\right), x_{n+1}-x^{*}\right)\right]=0 .
$$

Therefore, applying Lemma 2.12 to (3.42) we infer that $\lim _{n \rightarrow \infty}\left\|x_{n}-x^{*}\right\|=0$. This completes the proof.

Putting $M=N=1, F_{2} \equiv 0$, and $F_{1}=\Gamma$ an inverse-strongly monotone mapping on $C$ in Algorithm 3.1, we have the following algorithm.

Algorithm 3.2 Let $C$ be a nonempty closed convex subset of a real Hilbert space $H$, $\Theta: C \times C \rightarrow \mathbf{R}$ be a bifunction from satisfying conditions (A1)-(A4), $\varphi: C \rightarrow \mathbf{R} \cup\{+\infty\}$ be a proper lower semicontinuous and convex function with restriction (B1) or (B2), and $A: H \rightarrow H$ be $\mu_{0}$-inverse-strongly monotone. Let $R: C \rightarrow 2^{H}$ be a maximal monotone mapping, $B: C \rightarrow H$ be $\eta_{0}$-inverse-strongly monotone, $T: C \rightarrow C$ be a $\xi$-strictly pseudocontractive mapping, $S: H \rightarrow H$ is a nonexpansive mapping and $V: H \rightarrow H$ be a $\rho$-contraction with coefficient $\rho \in[0,1)$. Let $\Gamma: C \rightarrow H$ be $\zeta$-inverse-strongly monotone, and $F: H \rightarrow H$ be $\kappa$-Lipschitzian and $\eta$-strongly monotone with positive constants $\kappa, \eta>0$ such that $0 \leq \gamma<\tau$ and $0<\mu<\frac{2 \eta}{\kappa^{2}}$ where $\tau=1-\sqrt{1-\mu\left(2 \eta-\mu \kappa^{2}\right)}$. Assume that $\Omega:=\operatorname{GMEP}(\Theta, \varphi, A) \cap \mathrm{I}(B, R) \cap \operatorname{VI}(C, \Gamma) \cap \operatorname{Fix}(T) \neq \emptyset$. Let $\left\{\alpha_{n}\right\},\left\{\lambda_{n}\right\} \subset(0,1]$, $\left\{\beta_{n}\right\},\left\{\gamma_{n}\right\},\left\{\delta_{n}\right\} \subset[0,1],\left\{\rho_{n}\right\} \subset(0,2 \alpha],\left\{r_{n}\right\} \subset[c, d] \subset\left(0,2 \mu_{0}\right)$ and $\left\{\rho_{n}\right\} \subset[e, f] \subset\left(0,2 \eta_{0}\right)$. For arbitrarily given $x_{0} \in H$, let $\left\{x_{n}\right\}$ be a sequence generated by

$$
\left\{\begin{array}{l}
\Theta\left(u_{n}, y\right)+\varphi(y)-\varphi\left(u_{n}\right)+\left\langle A x_{n}, y-u_{n}\right\rangle+\frac{1}{r_{n}}\left\langle y-u_{n}, u_{n}-x_{n}\right\rangle \geq 0, \quad \forall y \in C, \\
v_{n}=J_{R, \rho_{n}}\left(I-\rho_{n} B\right) u_{n} \\
y_{n}=\beta_{n} x_{n}+\gamma_{n} P_{C}(I-v \Gamma) v_{n}+\delta_{n} T P_{C}(I-v \Gamma) v_{n}, \\
x_{n+1}=\lambda_{n} \gamma\left(\alpha_{n} V x_{n}+\left(1-\alpha_{n}\right) S x_{n}\right)+\left(I-\lambda_{n} \mu F\right) y_{n}, \quad \forall n \geq 0,
\end{array}\right.
$$

where $v \in(0,2 \zeta)$.

From Theorem 3.1, we have following result.

Corollary 3.1 In addition to assumption of Algorithm 3.2, suppose that

(i) $\lim _{n \rightarrow \infty} \lambda_{n}=0, \sum_{n=0}^{\infty} \lambda_{n}=\infty$ and $\lim _{n \rightarrow \infty} \frac{1}{\lambda_{n}}\left|1-\frac{\alpha_{n-1}}{\alpha_{n}}\right|=0$;

(ii) $\lim \sup _{n \rightarrow \infty} \frac{\alpha_{n}}{\lambda_{n}}<\infty, \lim _{n \rightarrow \infty} \frac{1}{\lambda_{n}}\left|\frac{1}{\alpha_{n}}-\frac{1}{\alpha_{n-1}}\right|=0$ and $\lim _{n \rightarrow \infty} \frac{1}{\alpha_{n}}\left|1-\frac{\lambda_{n-1}}{\lambda_{n}}\right|=0$;

(iii) $\lim _{n \rightarrow \infty} \frac{\left|\beta_{n}-\beta_{n-1}\right|}{\lambda_{n} \alpha_{n}}=0$ and $\lim _{n \rightarrow \infty} \frac{\left|\gamma_{n}-\gamma_{n-1}\right|}{\lambda_{n} \alpha_{n}}=0$; 
(iv) $\lim _{n \rightarrow \infty} \frac{\left|r_{n}-r_{n-1}\right|}{\lambda_{n} \alpha_{n}}=0$ and $\lim _{n \rightarrow \infty} \frac{\left|\rho_{n}-\rho_{n-1}\right|}{\lambda_{n} \alpha_{n}}=0$;

(v) $\beta_{n}+\gamma_{n}+\delta_{n}=1$ and $\left(\gamma_{n}+\delta_{n}\right) \xi \leq \gamma_{n}$ for all $n \geq 0$;

(vi) $\left\{\beta_{n}\right\} \subset[a, b] \subset(0,1)$ and $\liminf _{n \rightarrow \infty} \delta_{n}>0$.

Then

(a) $\lim _{n \rightarrow \infty} \frac{\left\|x_{n+1}-x_{n}\right\|}{\alpha_{n}}=0$

(b) $\omega_{w}\left(x_{n}\right) \subset \Omega$;

(c) $\left\{x_{n}\right\}$ converges strongly to a point $x^{*} \in \Omega$, which is a unique solution of HVIP (1.9), i.e.,

$$
\left\langle(\mu F-\gamma S) x^{*}, p-x^{*}\right\rangle \geq 0, \quad \forall p \in \Omega .
$$

Proof Since $F_{2} \equiv 0$ and $F_{1}=\Gamma$ a $\zeta$-inverse-strongly monotone mapping on $C$, it is easy to see that $\operatorname{GSVI}(G)=\operatorname{VI}(C, \Gamma)$. Thus, in terms of Theorem 3.1, we derive the desired result.

Putting $\Gamma=I-\Phi$, where $\Phi: C \rightarrow C$ is a $\xi_{0}$-strictly pseudocontractive mapping on $C$, in Algorithm 3.2, we obtain the following algorithm.

Algorithm 3.3 Let $C$ be a nonempty closed convex subset of a real Hilbert space $H$, $\Theta: C \times C \rightarrow \mathbf{R}$ be a bifunction satisfying conditions (A1)-(A4), $\varphi: C \rightarrow \mathbf{R} \cup\{+\infty\}$ be a proper lower semicontinuous and convex function with restriction (B1) or (B2), and $A: H \rightarrow H$ be $\mu_{0}$-inverse-strongly monotone. Let $R: C \rightarrow 2^{H}$ be a maximal monotone mapping, $B: C \rightarrow H$ be $\eta_{0}$-inverse-strongly monotone, $T: C \rightarrow C$ be a $\xi$-strictly pseudocontractive mapping, $S: H \rightarrow H$ is a nonexpansive mapping and $V: H \rightarrow H$ be a $\rho$-contraction with coefficient $\rho \in[0,1)$. Let $\Phi: C \rightarrow C$ be a $\xi_{0}$-strictly pseudocontractive mapping, and $F: H \rightarrow H$ be $\kappa$-Lipschitzian and $\eta$-strongly monotone with positive constants $\kappa, \eta>0$ such that $0 \leq \gamma<\tau$ and $0<\mu<\frac{2 \eta}{\kappa^{2}}$ where $\tau=1-\sqrt{1-\mu\left(2 \eta-\mu \kappa^{2}\right)}$. Assume that $\Omega:=\operatorname{GMEP}(\Theta, \varphi, A) \cap \mathrm{I}(B, R) \cap \operatorname{Fix}(\Phi) \cap \operatorname{Fix}(T) \neq \emptyset$. Let $\left\{\alpha_{n}\right\},\left\{\lambda_{n}\right\} \subset(0,1]$, $\left\{\beta_{n}\right\},\left\{\gamma_{n}\right\},\left\{\delta_{n}\right\} \subset[0,1],\left\{\rho_{n}\right\} \subset(0,2 \alpha],\left\{r_{n}\right\} \subset[c, d] \subset\left(0,2 \mu_{0}\right)$ and $\left\{\rho_{n}\right\} \subset[e, f] \subset\left(0,2 \eta_{0}\right)$. For arbitrarily given $x_{0} \in H$, let $\left\{x_{n}\right\}$ be a sequence generated by

$$
\left\{\begin{array}{l}
\Theta\left(u_{n}, y\right)+\varphi(y)-\varphi\left(u_{n}\right)+\left\langle A x_{n}, y-u_{n}\right\rangle+\frac{1}{r_{n}}\left\langle y-u_{n}, u_{n}-x_{n}\right\rangle \geq 0, \quad \forall y \in C, \\
v_{n}=J_{R, \rho_{n}}\left(I-\rho_{n} B\right) u_{n} \\
y_{n}=\beta_{n} x_{n}+\gamma_{n}(I-v(I-\Phi)) v_{n}+\delta_{n} T(I-v(I-\Phi)) v_{n}, \\
x_{n+1}=\lambda_{n} \gamma\left(\alpha_{n} V x_{n}+\left(1-\alpha_{n}\right) S x_{n}\right)+\left(I-\lambda_{n} \mu F\right) y_{n}, \quad \forall n \geq 0
\end{array}\right.
$$

where $v \in\left(0,1-\xi_{0}\right)$.

Corollary 3.2 In addition to assumption of Algorithm 3.3, suppose that

(i) $\lim _{n \rightarrow \infty} \lambda_{n}=0, \sum_{n=0}^{\infty} \lambda_{n}=\infty$ and $\lim _{n \rightarrow \infty} \frac{1}{\lambda_{n}}\left|1-\frac{\alpha_{n-1}}{\alpha_{n}}\right|=0$;

(ii) $\lim \sup _{n \rightarrow \infty} \frac{\alpha_{n}}{\lambda_{n}}<\infty, \lim _{n \rightarrow \infty} \frac{1}{\lambda_{n}}\left|\frac{1}{\alpha_{n}}-\frac{1}{\alpha_{n-1}}\right|=0$ and $\lim _{n \rightarrow \infty} \frac{1}{\alpha_{n}}\left|1-\frac{\lambda_{n-1}}{\lambda_{n}}\right|=0$;

(iii) $\lim _{n \rightarrow \infty} \frac{\left|\beta_{n}-\beta_{n-1}\right|}{\lambda_{n} \alpha_{n}}=0$ and $\lim _{n \rightarrow \infty} \frac{|| \gamma_{n}-\alpha_{n-1} \mid}{\lambda_{n} \alpha_{n}}=0$;

(iv) $\lim _{n \rightarrow \infty} \frac{\left|r_{n}-r_{n-1}\right|}{\lambda_{n} \alpha_{n}}=0$ and $\lim _{n \rightarrow \infty} \frac{\left|\rho_{n}-\rho_{n-1}\right|}{\lambda_{n} \alpha_{n}}=0$;

(v) $\beta_{n}+\gamma_{n}+\delta_{n}=1$ and $\left(\gamma_{n}+\delta_{n}\right) \xi \leq \gamma_{n}$ for all $n \geq 0$;

(vi) $\left\{\beta_{n}\right\} \subset[a, b] \subset(0,1)$ and $\liminf _{n \rightarrow \infty} \delta_{n}>0$.

Then

(a) $\lim _{n \rightarrow \infty} \frac{\left\|x_{n+1}-x_{n}\right\|}{\alpha_{n}}=0$ 
(b) $\omega_{w}\left(x_{n}\right) \subset \Omega$;

(c) $\left\{x_{n}\right\}$ converges strongly to a point $x^{*} \in \Omega$, which is a unique solution of HVIP (1.9), i.e.,

$$
\left\langle(\mu F-\gamma S) x^{*}, p-x^{*}\right\rangle \geq 0, \quad \forall p \in \Omega .
$$

Proof Since $\Phi: C \rightarrow C$ is a $\xi_{0}$-strictly pseudocontractive mapping on $C$, it is well known that for constant $\xi_{0} \in[0,1)$,

$$
\langle\Phi x-\Phi y, x-y\rangle \leq\|x-y\|^{2}-\frac{1-\xi_{0}}{2}\|(I-\Phi) x-(I-\Phi) y\|^{2}, \quad \forall x, y \in C .
$$

It is clear that in this case the mapping $\Gamma=I-\Phi$ is $\frac{1-\xi_{0}}{2}$-inverse-strongly monotone. Moreover, we have, for $v \in\left(0,1-\xi_{0}\right)$,

$$
\begin{aligned}
y_{n} & =\beta_{n} x_{n}+\gamma_{n} P_{C}(I-v \Gamma) v_{n}+\delta_{n} T P_{C}(I-v \Gamma) v_{n} \\
& =\beta_{n} x_{n}+\gamma_{n}(I-v(I-\Phi)) v_{n}+\delta_{n} T(I-v(I-\Phi)) v_{n} .
\end{aligned}
$$

Now let us show $\operatorname{Fix}(\Phi)=\operatorname{VI}(C, \Gamma)$. In fact, we have, for $\lambda>0$,

$$
\begin{aligned}
u \in \operatorname{VI}(C, \Gamma) & \Longleftrightarrow\langle\Gamma u, y-u\rangle \geq 0, \quad \forall y \in C \\
& \Longleftrightarrow\langle u-\lambda \Gamma u-u, u-y\rangle \geq 0, \quad \forall y \in C \\
& \Longleftrightarrow u=P_{C}(u-\lambda \Gamma u) \\
& \Longleftrightarrow u=P_{C}(u-\lambda u+\lambda \Phi u) \\
& \Longleftrightarrow\langle u-\lambda u+\lambda \Phi u-u, u-y\rangle \geq 0, \quad \forall y \in C \\
& \Longleftrightarrow\langle u-\Phi u, u-y\rangle \leq 0, \quad \forall y \in C \\
& \Longleftrightarrow u=\Phi u \\
& \Longleftrightarrow u \in \operatorname{Fix}(\Phi) .
\end{aligned}
$$

Consequently,

$$
\begin{aligned}
\Omega & =\operatorname{GMEP}(\Theta, \varphi, A) \cap \mathrm{I}(B, R) \cap \operatorname{VI}(C, \Gamma) \cap \operatorname{Fix}(T) \\
& =\operatorname{GMEP}(\Theta, \varphi, A) \cap \mathrm{I}(B, R) \cap \operatorname{Fix}(\Phi) \cap \operatorname{Fix}(T) .
\end{aligned}
$$

Therefore, by Corollary 3.1, we derive the desired result.

Remark 3.1 Our results generalize and improve results in $[12,20]$ and the references therein. 


\section{Author details}

'Department of Mathematics, Shanghai Normal University, Shanghai, 200234, China. ${ }^{2}$ Scientific Computing Key Laboratory of Shanghai Universities, Shanghai, 200234, China. ${ }^{3}$ Department of Mathematics, King Abdulaziz University, P.O. Box 80203, Jeddah, 21589, Saudi Arabia. ${ }^{4}$ Department of Mathematics, Aligarh Muslim University, Aligarh, India. ${ }^{5}$ Department of Mathematics and Statistics, King Fahd University of Petroleum and Minerals, Dhahran, Saudi Arabia. ${ }^{6}$ Center for Fundamental Science, Kaohsiung Medical University, Kaohsiung, Taiwan.

\section{Acknowledgements}

This article was funded by the Deanship of Scientific Research (DSR), King Abdulaziz University, Jeddah. Therefore, the authors acknowledge with thanks DSR, for technical and financial support.

\section{Received: 3 August 2014 Accepted: 16 October 2014 Published: 30 Oct 2014}

\section{References}

1. Ansari, QH, Lalitha, CS, Mehta, M: Generalized Convexity, Nonsmooth Variational Inequalities and Nonsmooth Optimization. CRC Press, Boca Raton (2013)

2. Oden, JT: Quantitative Methods on Nonlinear Mechanics. Prentice Hall, Englewood Cliffs (1986)

3. Ceng, L-C, Guu, S-M, Yao, J-C: Hybrid iterative method for finding common solutions of generalized mixed equilibrium and fixed point problems. Fixed Point Theory Appl. 2012, 92 (2012)

4. Korpelevich, GM: The extragradient method for finding saddle points and other problems. Matecon 12, 747-756 (1976)

5. Ceng, L-C, Ansari, QH, Yao, J-C: Relaxed extragradient iterative methods for variational inequalities. Appl. Math. Comput. 218, 1112-1123 (2011)

6. Ansari, QH: Metric Spaces: Including Fixed Point Theory and Set-Valued Maps. Narosa Publishing House, New Delhi (2014)

7. Almezel, S, Ansari, QH, Khamsi, MA: Topics in Fixed Point Theory. Springer, Cham (2014)

8. Peng, J-W, Yao, J-C: A new hybrid-extragradient method for generalized mixed equilibrium problems, fixed point problems and variational inequality problems. Taiwan. J. Math. 12, 1401-1432 (2008)

9. Verma, RU: On a new system of nonlinear variational inequalities and associated iterative algorithms. Math. Sci. Res. Hot-Line 3(8), 65-68 (1999)

10. Yamada, I: The hybrid steepest-descent method for the variational inequality problems over the intersection of the fixed-point sets of nonexpansive mappings. In: Batnariu, D, Censor, Y, Reich, S (eds.) Inherently Parallel Algorithms in Feasibility and Optimization and Their Applications, pp. 473-504. North-Holland, Amsterdam (2001)

11. Ceng, L-C, Yao, J-C: A hybrid iterative scheme for mixed equilibrium problems and fixed point problems. J. Comput. Appl. Math. 214, 186-201 (2008)

12. Cai, G, Bu, SQ: Strong and weak convergence theorems for general mixed equilibrium problems and variational inequality problems and fixed point problems in Hilbert spaces. J. Comput. Appl. Math. 247, 34-52 (2013)

13. Sahu, DR, Xu, H-K, Yao, J-C: Asymptotically strict pseudocontractive mappings in the intermediate sense. Nonlinear Anal. 70, 3502-3511 (2009)

14. Ceng, L-C, Wong, MM: Relaxed extragradient method for finding a common element of systems of variational inequalities and fixed point problems. Taiwan. J. Math. 17(2), 701-724 (2013)

15. Ceng, L-C, Wang, CY, Yao, J-C: Strong convergence theorems by a relaxed extragradient method for a general system of variational inequalities. Math. Methods Oper. Res. 67(3), 375-390 (2008)

16. Ansari, QH, Scahiable, S, Yao, J-C: System of vector equilibrium problems and its applications. J. Optim. Theory Appl. $107,547-557(2000)$

17. Ansari, QH, Yao, J-C: A fixed point theorem and its applications to the system of variational inequalities. Bull. Aust. Math. Soc. 59, 433-442 (1999)

18. Ansari, QH, Yao, J-C: Systems of generalized variational inequalities and their applications. Appl. Anal. 76(3-4), 203-217 (2000)

19. Lin, L-J, Yu, Z-T, Ansari, QH, Lai, L-P: Fixed point and maximal element theorems with applications to abstract economies and minimax inequalities. J. Math. Anal. Appl. 284, 656-671 (2003)

20. Yao, Y, Liou, YC, Marino, G: Two-step iterative algorithms for hierarchical fixed point problems and variational inequality problems. J. Appl. Math. Comput. 31(1-2), 433-445 (2009)

21. Ansari, QH, Ceng, L-C, Gupta, H: Triple hierarchical variational inequalities. In: Ansari, QH (ed.) Nonlinear Analysis: Approximation Theory, Optimization and Applications, pp. 231-280. Birkhäuser, Basel (2014)

22. Rockafellar, RT: Monotone operators and the proximal point algorithms. SIAM J. Control Optim. 14, 877-898 (1976)

23. Marino, G, Xu, HK: Weak and strong convergence theorems for strict pseudo-contractions in Hilbert spaces. J. Math. Anal. Appl. 329, 336-346 (2007)

24. Yao, Y, Liou, YC, Kang, SM: Approach to common elements of variational inequality problems and fixed point problems via a relaxed extragradient method. Comput. Math. Appl. 59, 3472-3480 (2010)

25. Huang, NJ: A new completely general class of variational inclusions with noncompact valued mappings. Comput. Math. Appl. 35(10), 9-14 (1998)

26. Ceng, L-C, Ansari, QH, Wong, MM, Yao, J-C: Mann type hybrid extragradient method for variational inequalities, variational inclusions and fixed point problems. Fixed Point Theory 13(2), 403-422 (2012)

27. Zeng, L-C, Guu, S-M, Yao, J-C: Characterization of H-monotone operators with applications to variational inclusions. Comput. Math. Appl. 50(3-4), 329-337 (2005)

28. Barbu, V: Nonlinear Semigroups and Differential Equations in Banach Spaces. Noordhoff, Groningen (1976)

29. Goebel, K, Kirk, WA: Topics on Metric Fixed Point Theory. Cambridge University Press, Cambridge (1990)

30. Xu, HK, Kim, TH: Convergence of hybrid steepest-descent methods for variational inequalities. J. Optim. Theory Appl. 119(1), 185-201 (2003) 
10.1186/1687-1812-2014-222

Cite this article as: Ceng et al.: Hybrid extragradient method for hierarchical variational inequalities. Fixed Point Theory and Applications 2014, 2014:222

Submit your manuscript to a SpringerOpen ${ }^{\circ}$ journal and benefit from:

- Convenient online submission

- Rigorous peer review

- Immediate publication on acceptance

Open access: articles freely available online

- High visibility within the field

- Retaining the copyright to your article

Submit your next manuscript at $\boldsymbol{s p r i n g e r o p e n . c o m ~}$ 